\title{
Different activation signals induce distinct mast cell degranulation strategies
}

\author{
Nicolas Gaudenzio, ${ }^{1,2}$ Riccardo Sibilano, ${ }^{1,2}$ Thomas Marichal, ${ }^{3}$ Philipp Starkl, ${ }^{1,2}$ Laurent L. Reber, ${ }^{1,2}$ Nicolas Cenac, ${ }^{4}$ \\ Benjamin D. McNeil, ${ }^{5}$ Xinzhong Dong, ${ }^{5,6}$ Joseph D. Hernandez, ${ }^{1,2}$ Ronit Sagi-Eisenberg, ${ }^{7}$ Ilan Hammel, ${ }^{8}$ Axel Roers, ${ }^{9}$ \\ Salvatore Valitutti, ${ }^{4}$ Mindy Tsai, ${ }^{1,2}$ Eric Espinosa, ${ }^{4}$ and Stephen J. Galli ${ }^{1,2,10}$ \\ 'Department of Pathology, Stanford University School of Medicine, Stanford, California, USA. ${ }^{2}$ Sean N. Parker Center for Allergy and Asthma Research, Stanford University School of Medicine, Stanford \\ California, USA. ${ }^{3}$ GICA-Research and Faculty of Veterinary Medicine, University of Liège, Liège, Belgium. ${ }^{4}$ INSERM, U1043, Toulouse, France. ${ }^{5 T h e}$ Solomon H. Snyder Department of Neuroscience, \\ Center for Sensory Biology, and ${ }^{6}$ Howard Hughes Medical Institute, Johns Hopkins University, School of Medicine, Baltimore, Maryland, USA. 'Department of Cell and Developmental Biology and \\ ${ }^{8}$ Department of Pathology, Sackler Faculty of Medicine, Tel-Aviv University, Ramat Aviv, Tel Aviv, Israel. ${ }^{9}$ Institute for Immunology, University of Technology Dresden, Medical Faculty Carl-Gustav Carus, \\ Dresden, Germany. ${ }^{10}$ Department of Microbiology and Immunology, Stanford University School of Medicine, Stanford, California, USA.
}

\begin{abstract}
Mast cells (MCs) influence intercellular communication during inflammation by secreting cytoplasmic granules that contain diverse mediators. Here, we have demonstrated that MCs decode different activation stimuli into spatially and temporally distinct patterns of granule secretion. Certain signals, including substance $P$, the complement anaphylatoxins C3a and C5a, and endothelin 1, induced human MCs rapidly to secrete small and relatively spherical granule structures, a pattern consistent with the secretion of individual granules. Conversely, activating MCs with anti-IgE increased the time partition between signaling and secretion, which was associated with a period of sustained elevation of intracellular calcium and formation of larger and more heterogeneously shaped granule structures that underwent prolonged exteriorization. Pharmacological inhibition of IKK- $\beta$ during IgE-dependent stimulation strongly reduced the time partition between signaling and secretion, inhibited SNAP23/STX4 complex formation, and switched the degranulation pattern into one that resembled degranulation induced by substance $P$. IgE-dependent and substance $P$-dependent activation in vivo also induced different patterns of mouse MC degranulation that were associated with distinct local and systemic pathophysiological responses. These findings show that cytoplasmic granule secretion from MCs that occurs in response to different activating stimuli can exhibit distinct dynamics and features that are associated with distinct patterns of MC-dependent inflammation.
\end{abstract}

\section{Introduction}

Secretory granule exocytosis is a tightly regulated process, shared by mast cells (MCs) and other eukaryotic cells, that influences the outcome of diverse physiological and pathological processes (1). MC degranulation can contribute to resistance to venoms (2-4), bacteria (5), and parasites $(6,7)$ but also to the morbidity and mortality associated with allergic diseases $(8,9)$. Aggregation of the high-affinity IgE receptor (FceRI) on the MC plasma membrane, induced when specific antigens cross-link FceRI-bound IgE, activates a complex intracellular signaling pathway resulting in secretion of cytoplasmic granule content into the extracellular environment (10), which can orchestrate local or systemic inflammation (11-16). However, stimuli that can activate MCs via various receptors that are distinct from those binding antibodies also can contribute to inflammatory processes (17-19). Examples of such stimuli include complement anaphylatoxins (e.g., C3a and C5a)

\section{Related Commentary: p. 3735}

Authorship note: R. Sibilano and T. Marichal contributed equally to this work. Conflict of interest: The authors have declared that no conflict of interest exists. Submitted: November 13, 2015; Accepted: August 12, 2016. Reference information: / Clin Invest. 2016;126(10):3981-3998. doi:10.1172/JCI85538.
(19), the vasoconstrictor peptide endothelin 1 (ET1) (17), and a panel of cationic substances such as the neuropeptide substance $P$ (SP) (20) and drugs associated with pseudoallergic reactions (e.g., icatibant and cetrorelix) $(21,22)$.

Although important progress has been made in the analysis of MC degranulation in situ (23-28), technical constraints have limited the spatiotemporal resolution of this process, which has hampered analysis of the dynamics and quantitative characteristics of granule exteriorization in real time at the single-cell level. We developed a dynamic imaging system that, in contrast to static structural imaging (such as conventional transmission electron microscopy [TEM]), can follow in real time the spatially complex, rapidly evolving features of MCs undergoing activation. By combining newly designed granule detection and modeling techniques, we demonstrate that both human primary MCs in vitro and mouse dermal MCs in vivo can respond to distinct stimuli of activation by finely regulating the dynamics and features of MC granule secretion.

\section{Results}

MCs differentially exteriorize secretory granules in response to different stimuli. We compared MC responses to (a) SP, an endogenous cationic 11-amino acid neuropeptide implicated in various 

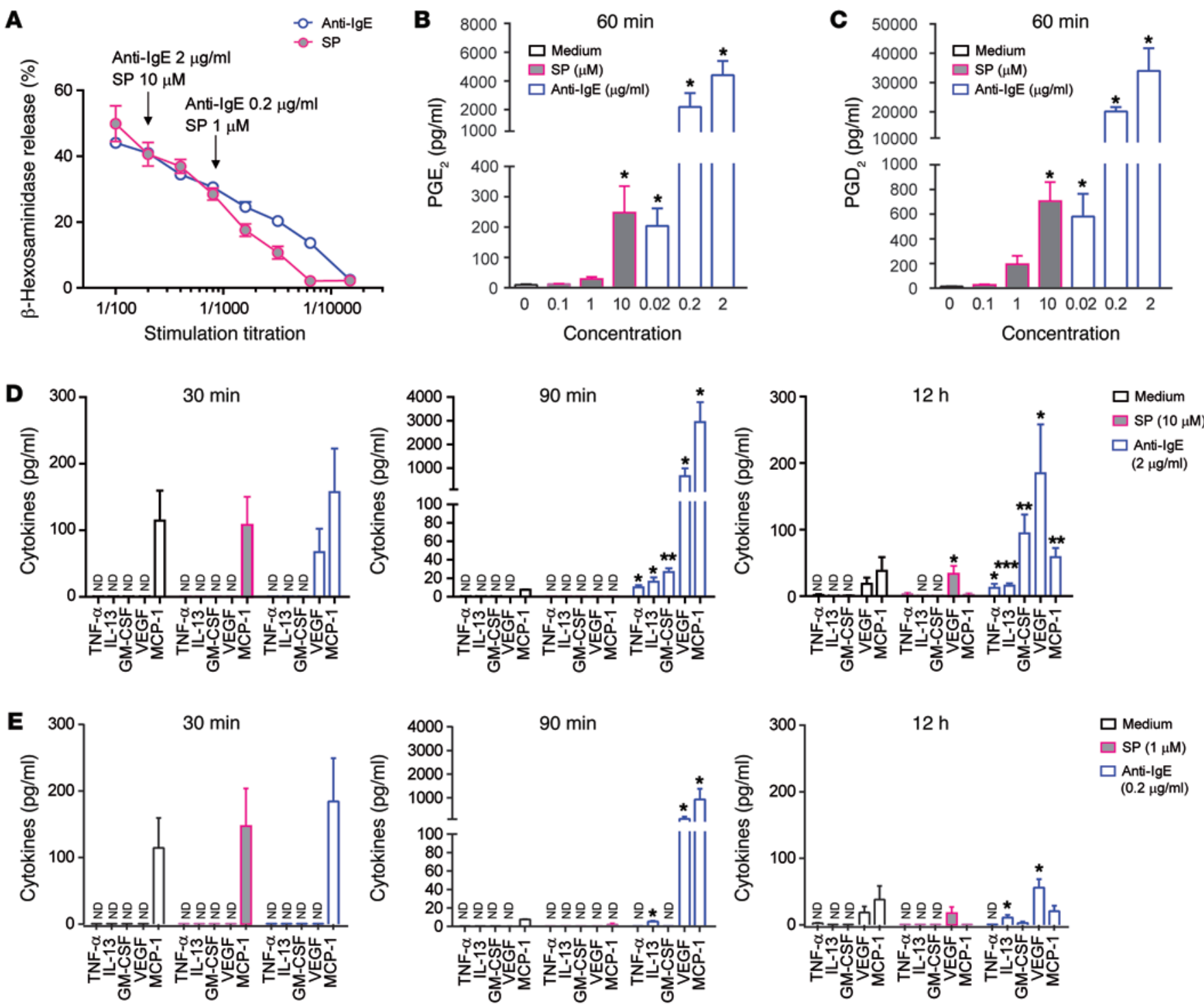

Figure 1. Human MC activation by SP or anti-IgE induces different patterns of secretion of lipid mediators, cytokines, and chemokines. IgE-sensitized or nonsensitized PBCMCs were incubated in the presence of anti-IgE (blue) or SP (pink), respectively, or with medium alone (no stimulation, black). (A) Percentage of $\beta$-hexosaminidase release 60 minutes after stimulation with different concentrations of stimulatory molecules. (B and $\mathbf{C}$ ) Production of prostaglandin $\mathrm{E}_{2}\left(\mathrm{PGE}_{2}\right)$ (B) or $\mathrm{PGD}_{2}$ (C) 60 minutes after addition of $0.1,1$, or $10 \mu \mathrm{M} \mathrm{SP}$ or $0.02,0.2$, or $2 \mu \mathrm{g} / \mathrm{ml}$ of anti-lgE or medium alone. (D and $\mathbf{E}$ ) Production of TNF- $\alpha$, IL-13, GM-CSF, VEGF, and MCP-1 30 minutes (left panel), 90 minutes (middle panel), or 12 hours (right panel) after addition of $10 \mu M$ SP or $2 \mu \mathrm{g} / \mathrm{ml}$ of anti-IgE or medium alone (D) or $1 \mu \mathrm{M} \mathrm{SP}$ or $0.2 \mu \mathrm{g} / \mathrm{ml}$ of anti-IgE or medium alone (E). Mean \pm SEM; 2 -tailed, unpaired $t$ test (vehicle vs. SP or vehicle vs. DNP); ${ }^{*}<0.05 ;{ }^{* *} P<0.01$; ${ }^{* *} P<0.001$; nd, not detected. Data are pooled from 5 independent experiments performed with PBCMCs from 4 different donors, all of which gave similar results.

inflammatory conditions $(18,29,30)$ and a strong activator of the receptor MRGPRX2 (the ortholog of MRGPRB2: the receptor for cationic secretagogues in the mouse) (30-32), and (b) an antibody-dependent stimulus, anti-IgE, which activates IgE-bearing MCs by cross-linking FceRI-bound IgE. Similar levels of degranulation of primary human peripheral blood-derived cultured MCs (PBCMCs) $(33,34)$, measured by release of the granule-stored mediator $\beta$-hexosaminidase, were induced when PBCMCs were stimulated with $2 \mu \mathrm{g} / \mathrm{ml}$ of anti-IgE or with $10 \mu \mathrm{M}$ SP (Figure 1A). Except as otherwise noted, we used these conditions of stimulation for all subsequent studies analyzing PBCMC activation via FceRI or MRGPRX2. While anti-IgE stimula- tion dose-dependently induced strong de novo secretion of lipid mediators (e.g., prostaglandins $\mathrm{D}_{2}$ and $\mathrm{E}_{2}$ ) and several inflammatory cytokines and chemokines, SP triggered secretion of only low amounts of lipid mediators and VEGF (Figure 1, B-E). Thus, when we used SP or anti-IgE under conditions that resulted in the same extent of PBCMC degranulation, activation via MRGPRX2 versus FceRI triggered distinct patterns of secretion of MC mediators not stored in the granules.

To characterize the spatiotemporal features of MC degranulation and the early steps of granule exocytosis, we used soluble fluorochrome-labeled avidin (sulforhodamine 101-coupled avidin [Av.SRho]), a method we recently described (34). During 
MC degranulation, which results in fusion of secretory granule membranes with the plasma membrane (35), the granule matrix is externalized and rapidly bound by the fluorescent probe, allowing monitoring, by high-resolution confocal microscopy, of degranulation dynamics in real time and in single cells (Supplemental Figure 1; supplemental material available online with this article; doi:10.1172/JCI85538DS1).

PBCMCs were loaded with Fluo- 4 and stimulated in the presence of Av.SRho to monitor early $\left[\mathrm{Ca}^{2+}\right]_{\mathrm{i}}$ signaling and degranulation dynamics, respectively (Figure 2, A-H, and Supplemental Video 1). Anti-IgE-mediated PBCMC activation triggered a strong and sustained increase in $\left[\mathrm{Ca}^{2+}\right]_{i}$ (Figure 2, A,C, and E) followed by progressive degranulation over 30 minutes (Figure 2, A, F, and $\mathrm{H}$ ). By contrast, SP stimulation mobilized a rapid but brief increase in $\left[\mathrm{Ca}^{2+}\right]_{\mathrm{i}}$ (Figure 2, B, D, and E) followed by much faster degranulation (Figure 2, B, G, and H). Anti-IgE-stimulated PBCMCs exhibited a lag time $(\Delta \mathrm{T})$ of about 5 minutes between the early increase in $\left[\mathrm{Ca}^{2+}\right]_{\mathrm{i}}$ and detection of the first budding extracellular granule structures, but this $\Delta \mathrm{T}$ was markedly shortened (almost absent in some experiments) in SP-stimulated MCs (Figure 2, I-K). Timelapse flow cytometry analyses of degranulation in PBCMCs indicated that the observed distinct degranulation dynamics were not affected by changing of the strength of stimulation (Supplemental Figure 2, A and B).

In accord with previous reports $(30,32,36)$, we confirmed, using shRNA treatment of PBCMCs, that SP-induced MC degranulation, but not anti-IgE-induced degranulation, was mediated by activation of the receptor MRGPRX2 (Supplemental Figure 3). We also tested 3 other cationic agonists of MRGPRX2 (compound 48/80, icatibant, and cetrorelix; refs. 21, 22, 32, 37), and these molecules exhibited comparable abilities to induce PBCMC activation with an "SP-like" pattern of degranulation, suggesting that this is an intrinsic feature of MRGPRX2-mediated MC activation (Supplemental Figure 4).

We also analyzed the degranulation dynamics and $\Delta \mathrm{T}$ of single PBCMCs activated either by IgG immune complexes (mouse anti-dinitrophenyl [anti-DNP] IgG and DNP-human serum albu$\min$ [DNP-HSA]) (34) or by additional stimuli that can activate MCs by binding to various $\mathrm{G}$ protein-coupled receptors (GPCRs) (i.e., C5a, C3a, and ET1). PBCMCs activated by C5a, C3a, or ET1 exhibited fast degranulation, with a $\Delta \mathrm{T}$ of approximately 1 minute, very similar to that observed after stimulation with SP (Supplemental Figure 5, C-H and K, and Supplemental Figure 6, A-F and I). The PBCMC degranulation induced by mouse IgG immune complexes was modest in amount compared with that induced by the other stimuli tested (Supplemental Figure 5, A and B) but exhibited a delayed and progressive pattern (Supplemental Figure 5, I-K, and Supplemental Figure 6, G-I) resembling that induced by anti-IgE (Figure 2, A, F, and I). These data indicate that MCs can respond to activation stimuli with at least 2 different patterns of degranulation: the delayed and progressive pattern seen in response to stimulation with anti-IgE or IgG immune complexes and the more rapid process observed in MCs stimulated with cationic agonists (i.e., SP, compound 48/80, icatabant, and cetrorelix), C3a, C5a, or ET1. These findings indicate that the time of transport of MC cytoplasmic granules to the plasma membrane can depend on the nature of the activation signal.
MCs differentially mobilize intracellular secretory granules for exteriorization in response to different stimuli. The variability we observed in the $\Delta \mathrm{T}$ between early $\left[\mathrm{Ca}^{2+}\right]_{\mathrm{i}}$ signaling and detection of the first Av.SRho ${ }^{+}$exteriorized granule structures in MCs stimulated with either of the 2 antibody-dependent stimuli versus with any of the other stimuli tested (Figure 2, I-K, and Supplemental Figure 6) prompted us to investigate whether MCs could differentially regulate the mobilization of intracellular granules before full exteriorization of the granule content. Prior TEM studies of human MCs showed that, during IgE-dependent MC degranulation, individual cytoplasmic granules fused with each other and with the plasma membrane, resulting in the exteriorization of the matrices of multiple individual granules via single openings to the exterior, a process called compound exocytosis $(38,39)$. Moreover, based on her TEM observations, Ann Dvorak and colleagues proposed that individual cytoplasmic granules of human MCs activated by anti-IgE could undergo fusion with each other even before such fused granules formed a communication with the exterior via fusion of their granule membrane with the plasma membrane $(38,39)$. Yet TEM studies are not well suited to detect very transient communications between the granules and the plasma membrane, such as might occur during "kiss-and-run" fusion (40).

Fluorescence dequenching of previously endocytosed dextran-FITC (resulting in increased fluorescence intensity) can be used to monitor the increase in $\mathrm{pH}$ occurring when the cytoplasmic granules of rat basophil leukemia (RBL) cells fuse with the plasma membrane $(26,41)$. This method has been reported to detect FITC dequenching caused by full fusion of granules with the plasma membrane, kiss-and-run transient fusion, or compound exocytosis (26). We chose this approach to investigate granule-plasma membrane fusion events in otherwise unmanipulated single human MCs, thereby avoiding the need to use cells that had been engineered to overexpress particular vesicular markers potentially involved in exocytosis (e.g., CD63).

We found that the FITC fluorescence signal was stable and constant over time in nonstimulated human PBCMCs (Figure 3, $\mathrm{A}$ and $\mathrm{B}$, and Supplemental Video 2), but that anti-IgE-induced activation caused this signal to increase significantly during the $\Delta \mathrm{T}$ period, i.e., before detection of the first externalized granules by binding of Av.SRho (Figure 3, C, D, and G, and Supplemental Video 2). We also observed similar degranulation dynamics and a transient increase in FITC fluorescence signal using dextran-FITCloaded mouse peritoneal cell-derived MCs $(42,43)$ that had been activated with IgE and specific antigen (Supplemental Figure 7).

By contrast, stimulation with SP resulted in the nearly immediate exocytosis of dextran-FITC-labeled granule structures (together with the rapid detection of exteriorized $\mathrm{Av} . \mathrm{SRho}^{+}$granule structures) without any prior increase in dextran-FITC fluorescence intensity (Figure 3, E-G, and Supplemental Video 2). We also found that C5a, ET1, and C3a each resulted in "SP-like" fast exocytosis of dextran-FITC-preloaded granule structures (Supplemental Figure 8, A-F and I, and Supplemental Video 3). However, stimulation of PBCMCs with IgG immune complexes triggered a response similar to that observed with anti-IgE, i.e., an initial increase in dextran-FITC fluorescence intensity followed by a slower rate of granule exo- 
A

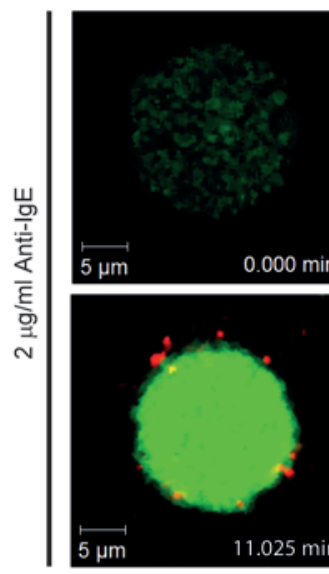

B
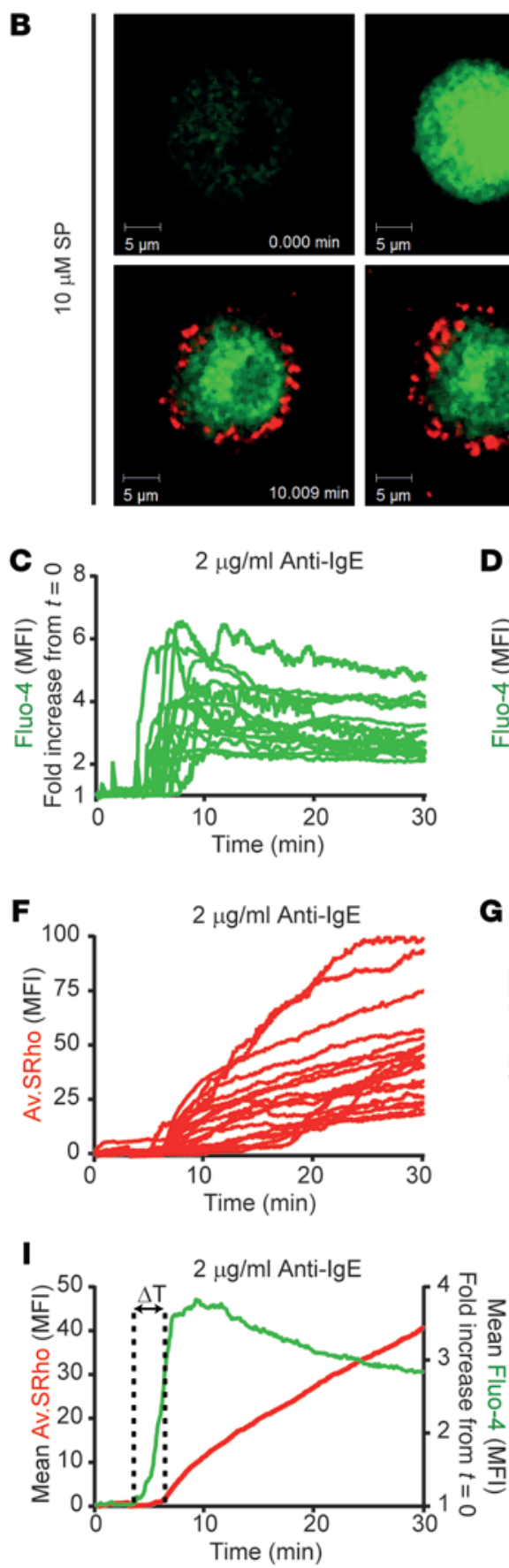
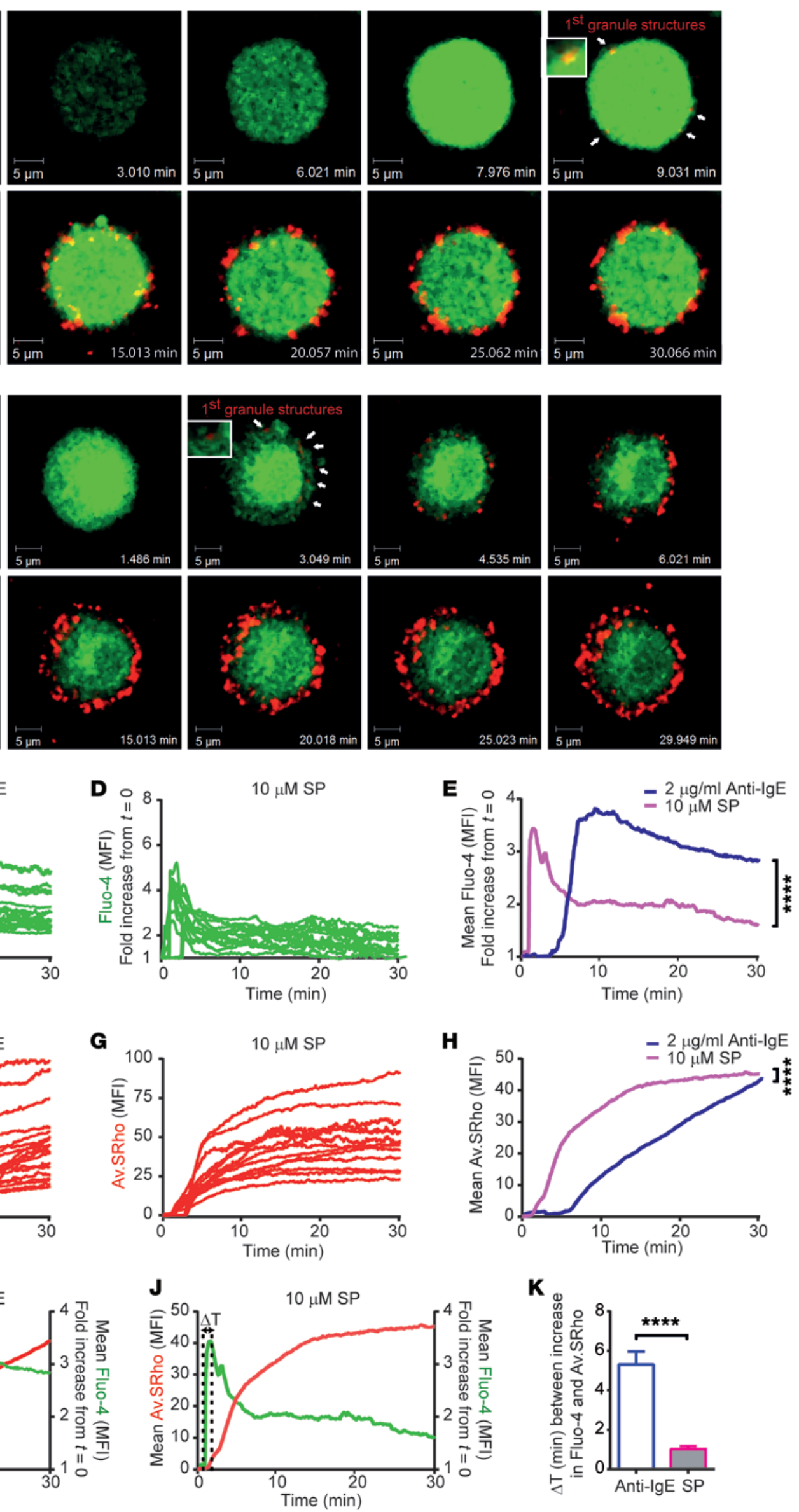
Figure 2. Human MC activation by SP or anti-lgE induces distinct $\left[\mathrm{Ca}^{2+}\right]_{\mathrm{i}}$ signaling and degranulation dynamics. IgE-sensitized or nonsensitized PBCMCs were loaded with Fluo-4 and stimulated with anti-IgE antibodies or SP in the presence of Av.SRho. Fluo-4 (green, $\left[\mathrm{Ca}^{2+}\right]_{i}$ ) and Av.SRho (red, identifying exteriorized granule structures) fluorescence was measured, at the single-cell level, using time-lapse confocal microscopy in a controlled atmosphere $\left(37^{\circ} \mathrm{C}\right.$ and $\left.5 \% \mathrm{CO}_{2}\right)$. (A) Representative time-lapse of a single lgE-sensitized PBCMC activated with anti-lgE. (B) Representative time-lapse of a single PBCMC activated with SP. (A and B) Scale bars: $5 \mu \mathrm{m}$; white insets show a budding granule structure at higher magnification; arrows indicate first budding granule structures; time scale reflects the kinetics of the responses induced by the 2 stimuli. (C and D) Single-cell analyses of Fluo-4 mean fluorescence intensity (MFI) following anti-IgE (C) or SP (D) stimulation. (E) Mean curves of Fluo-4 MFI following anti-IgE (blue) or SP (pink) stimulation. (F and $\mathbf{G}$ ) Single-cell analyses of Av.SRho MFI following anti-lgE (F) or SP (G) stimulation. (H) Mean curves of Av.SRho MFI following anti-IgE (blue) or SP (pink) stimulation. Mean; 2-way ANOVA; ${ }^{* * *} P<0.0001$. Data are from the 3 independent experiments performed, each of which gave similar results. (I and J) Mean curves of Fluo-4 and Av.SRho MFI following anti-lgE (I) or SP (J) stimulation; dotted lines and arrows indicate the lag time $(\Delta T)$ measured between the increase in $\left[\mathrm{Ca}^{2+}\right]_{i}$ and the detection of the first budding granule structures. (K) Mean $\Delta \mathrm{T}$ measured following anti-lgE (blue) or SP (pink) stimulation. Mean \pm SEM; 2 -tailed, unpaired $t$ test; ${ }^{* * * *} P<0.0001$. Data are from 3 independent experiments performed with PBCMCs from 3 donors (at least 30 single PBCMCs analyzed per condition), each of which gave similar results.

cytosis (Supplemental Figure 8, G-I, and Supplemental Video $3)$. These observations indicate that, compared with MCs activated with SP, C3a, C5a, or ET1 (in which no FITC dequenching is detected, probably because the granules are rapidly released and the dextran-FITC rapidly diffuses away; ref. 26), MCs activated by antibody-dependent mechanisms exhibit a prolonged interval between the first evidence of cytoplasmic granule deacidification (which probably reflects at least transient fusion of the granule membranes with the plasma membrane; refs. 26, 41) and the first appearance of detectable Av.SRho ${ }^{+}$ granule matrix content at the cell surface. TEM studies of PBCMCs derived from 2 donors showed that some of the PBCMCs activated with anti-IgE exhibited evidence of compound exocytosis, with granule matrix material exposed to the cell exterior, as early as 3 minutes after stimulation (Supplemental Figure 9A). This finding might reflect a relatively rapid pattern of anti-IgE-induced degranulation in these 2 PBCMC populations and/or indicate that there may be a delay between the first appearance of granule matrix material at the cell surface as detected by TEM and the binding of sufficient Av.SRho to such structures to render them detectably Av.SRho ${ }^{+}$(which is first observed in most $\mathrm{PBCMC}$ populations at $\sim 5$ minutes after anti-IgE stimulation). By contrast, $\mathrm{PBCMCs}$ stimulated with SP exhibited exteriorized granule structures, but few images suggestive of compound exocytosis.

$M C s$ activated with anti-IgE versus SP exhibit differences in intracellular signaling. Activation of FceRI triggers a complex cascade of intracellular tyrosine kinases that induce membrane fusion events regulated by soluble $N$-ethylmaleimide-sensitive factor attachment protein receptors (SNAREs), acting in synergy with other accessory proteins $(11-14,16,44)$. However, the signaling events and SNARE components involved in GPCRdependent MC degranulation are not as well understood. We compared mobilization of key signaling molecules in human
MCs activated with anti-IgE versus SP, focusing on some of those known to influence $\left[\mathrm{Ca}^{2+}\right]_{\mathrm{i}}$ levels and $\mathrm{MC}$ degranulation: the serine-threonine kinase AKT, protein kinase $\mathrm{C}$ (PKC), and the inhibitor of $\mathrm{NF}-\kappa \mathrm{B}$ kinase subunit $\beta$ (IKK- $\beta$ ). In the 6 different PBCMC populations tested, we detected AKT, PKC, and IKK- $\alpha / \beta$ phosphorylation largely or fully following stimulation with anti-IgE but not SP (Figure $3 \mathrm{H}$ ), indicating that SP can induce $\mathrm{PBCMC}$ degranulation independently of activation of the canonical AKT, PKC, and IKK- $\beta$ signaling pathway. We found that compound 48/80, C3a, C5a, and ET1 also did not induce detectable IKK- $\alpha / \beta$ phosphorylation (data not shown).

To investigate whether human MCs activated by anti-IgE versus SP differentially mobilized SNARE partners, we used coimmunoprecipitation employing antibodies whose specificity we confirmed (Supplemental Figure 10) to assess complex formation between synaptosomal-associated protein-23 (SNAP23) and syntaxin-4 (STX4), which has been identified as a key step involved in MC compound exocytosis (44-47). SP-mediated activation failed to trigger the formation of such complexes, whereas anti-IgE stimulation did so (Figure 3I). By contrast, we confirmed an association between MUNC-18-2 and STX3 in unstimulated MCs (28), which appeared to increase slightly in MCs stimulated with either SP or anti-IgE (Figure 3J). This suggests that MUNC-18-2/STX3 interactions, which recently have been implicated in membrane fusion events (28), might be important for those membrane fusion events shared by human MCs responding to activation by anti-IgE or SP, such as heterotypic fusion between the granule and plasma membranes. Taken together, our results indicate that MCs can use distinct degranulation strategies when stimulated via FceRI or MRGPRX2 and that substantial activation of the AKT/PKC/IKK- $\beta$ pathway and SNAP23/STX4 complex formation are features of FceRI-dependent activation of human MCs that are not shared with SP-dependent MC activation.

MC IKK- $\beta$ activity is critical for the anti-IgE-induced pattern of $M C$ degranulation. IKK- $\beta$ activation is considered to represent an essential step for IgE-mediated MC cytokine secretion, but the possible roles of IKK- $\beta$ in MC degranulation are unsettled $(47,48)$. Inhibition of IKK- $\beta$ kinase activity with BMS-345541, which has been reported to be a highly selective inhibitor of the 2 catalytic subunits of IKK- $\beta$ (49-53), did not interfere with the extent of antiIgE-induced MC granule exteriorization at 30 minutes (Figure 4, A-C, and Supplemental Video 4). However, compared with results in control MCs treated with medium containing DMSO alone, treatment with BMS-345541 resulted in the development of only a brief increase in $\left[\mathrm{Ca}^{2+}\right]_{\mathrm{i}}$ followed by a much faster degranulation process, with a statistically significant reduction in the $\Delta \mathrm{T}$ between signaling and secretion (from $\sim 3$ minutes for the DMSO-treated PBCMCs to 1.8 minutes for the BMS-345541-treated cells) (Figure 4, A-D, and Supplemental Video 4). Finally, while anti-IgE-induced IKK- $\beta$ phosphorylation was not affected by BMS-345541 treatment (Figure 4E), SNAP23/STX4 complex formation (Figure 4F), dequenching of FITC in dextran-FITC-labeled cytoplasmic granules (Figure 4, G-J, and Supplemental Video 5), and production of cytokines (Figure $4 \mathrm{~K})$ were each significantly diminished. Taken together, these findings show that treatment with BMS-345541 in effect switched the features of the typical spatiotemporal pattern of degranulation seen in MCs stimulated with anti-IgE to those seen with activation by SP. 
A

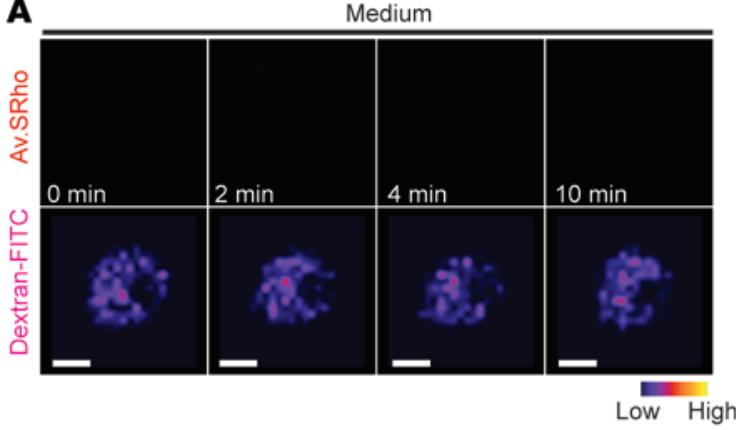

C

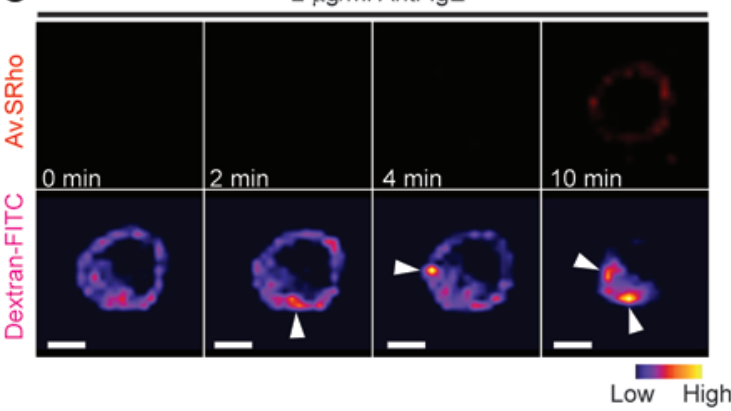

E

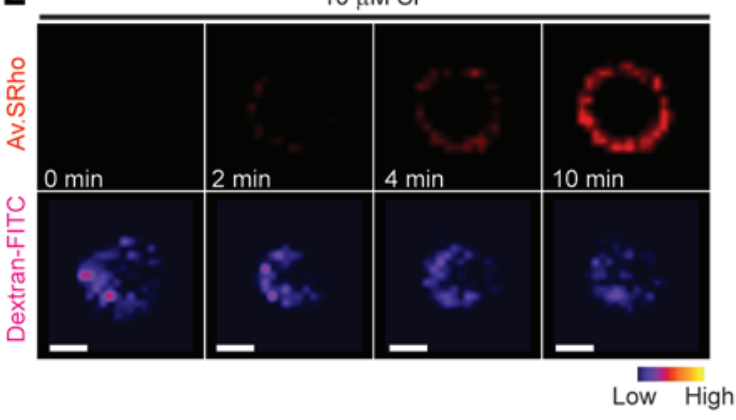

B

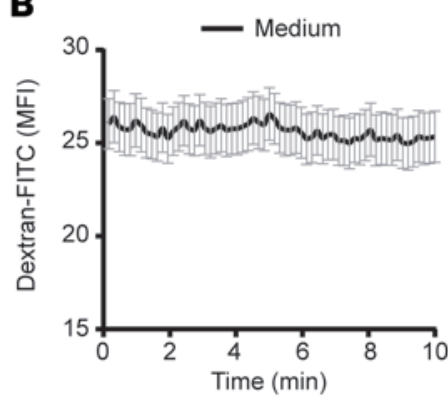

G

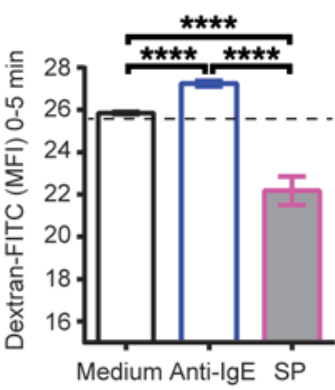

D

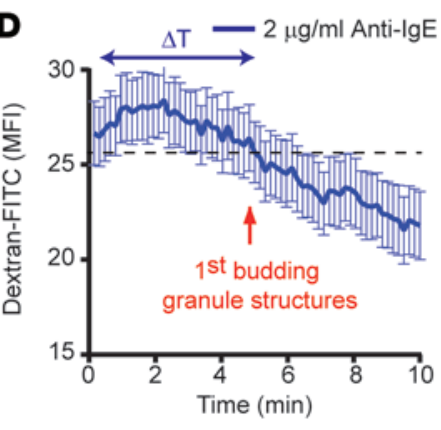

H
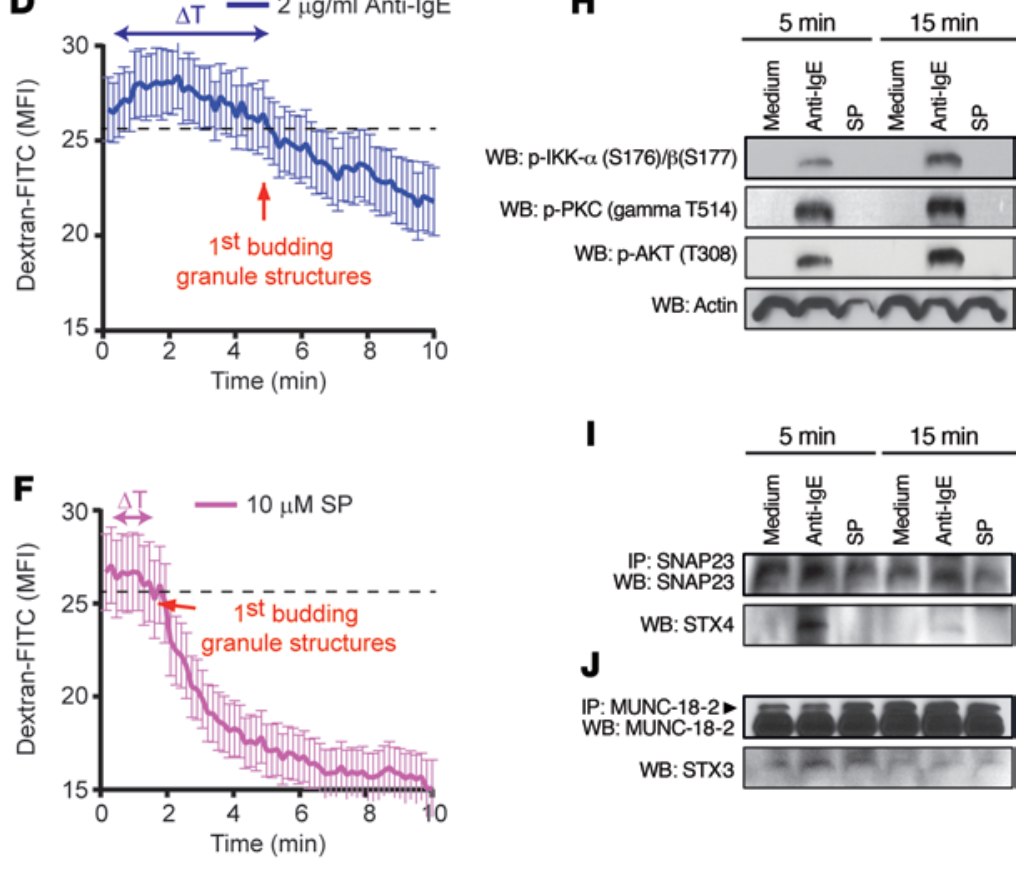

I

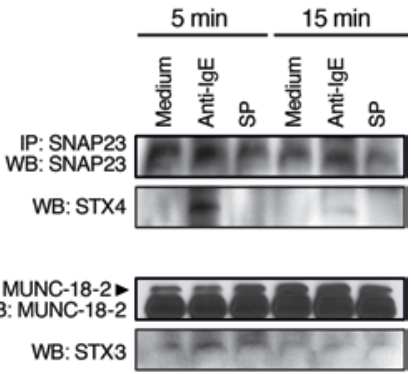

Figure 3. Signaling events and fluorescence dequenching patterns in dextran-FITC-labeled cytoplasmic granules in MCs stimulated via Fc\&RI or MRGPRX2. (A-F) IgE-sensitized or nonsensitized dextran-FITC-loaded PBCMCs were stimulated with $2 \mu \mathrm{g} / \mathrm{ml}$ of anti-lgE (blue) or $10 \mu \mathrm{M}$ SP (pink), respectively, or with medium alone (black), in the presence of Av.SRho. (A) Representative time-lapse sequence of Av.SRho (upper panels, red) and dextran-FITC (lower panels, pseudocolor) in a PBCMC incubated with medium alone. (B) Mean curve of pooled single-cell analyses of dextran-FITC MFI in PBCMCs incubated with medium. (C-F) Same experiment as in A and B in PBCMCs stimulated with anti-lgE (white arrowheads indicate increases in dextran-FITC fluorescence) or SP. Scale bars: $5 \mu \mathrm{m}$. (C) Average of dextran-FITC MFI between $t=0$ and $t=5$ minutes. (H-J) IgE-sensitized or nonsensitized PBCMCs were stimulated for 5 or 15 minutes with $2 \mu \mathrm{g} / \mathrm{ml}$ of anti-IgE or $10 \mu \mathrm{M}$ SP or with medium alone. (H) Expression of phospho-IKK- $\alpha / \beta$, phospho-PKC, and phospho-AKT in PBCMC lysates. Actin was used as loading control. (I) Immunoprecipitation of SNAP23/STX4 complexes in resting PBCMCs or activated with $2 \mu \mathrm{g} / \mathrm{ml}$ of anti-lgE or $10 \mu \mathrm{M}$ SP. Immunoprecipitated SNAP23 was resolved and probed for STX4. (J) Immunoprecipitation of MUNC-18/STX3 complexes performed for the same times and conditions tested in I. Immunoprecipitated MUNC-18-2 was resolved and probed for STX3. Mean \pm SEM; 2-tailed, unpaired $t$ test; ${ }^{* * * *} P<0.0001$. Data in A-G are from 4 independent experiments with PBCMCs from 2 donors ( 35 single PBCMCs analyzed per condition); data in $\mathbf{H}$ and $\mathbf{I}$ are representative of those obtained in 6 independent experiments, each of them performed with PBCMCs from a different single donor, all of which gave similar results.

These results support the hypothesis that IKK- $\beta$ activation and SNAP23/STX4 complex formation represent important components of the signaling that helps to orchestrate the compound exocytosis degranulation pattern observed in anti-IgEstimulated human MCs.

The size, number, and shape of MC exteriorized granule structures can vary depending on the nature of the activation stimulus. We next investigated whether the distinct patterns of human MC degranulation seen in response to anti-IgE or SP might also be associated with the exteriorization of granule structures exhibit- ing distinct physical properties. We developed a new 3D granule visualization approach based on use of a computer-generated representation of Av.SRho fluorescence (Supplemental Figure 11) and then precisely calculated the volume, number, and shape of such modeled exocytosed Av.SRho ${ }^{+}$granule structures. Such Av.SRho ${ }^{+}$granule structures might consist of either the matrices contained in single cytoplasmic granules of various sizes (now free of their granule membranes as a result of the fusion of the granule membranes with the plasma membrane during degranulation) or single structures consisting of the combined 
matrices of 2 or more individual granules (e.g., as formed by fusion between/among the membranes of individual granules during compound exocytosis, or after their individual secretion). Because extensive evidence indicates that MC cytoplasmic granules can increase progressively in size over time, particularly in MCs not undergoing activation for degranulation (54), measurements of volume alone cannot distinguish between a single granule matrix or an aggregate of 2 or more granule matrices. However, prior TEM observations indicate that single granules are more likely to approach a spherical shape than would aggregates of multiple granule matrices $(38,39)$. We therefore used our 3D modeling approach to measure the number, volume, and sphericity of exocytosed Av.SRho ${ }^{+}$granule structures produced by anti-IgE- or SP-activated MCs at multiple time points during the degranulation process (Figure 5A and Supplemental Video 6).

We first compared the total accumulated volume of exocytosed granule structures (i.e., total amount of externalized Av.SRho ${ }^{+}$ material per MC) during the first 60 seconds of degranulation. Because of the long $\Delta \mathrm{T}$ before the onset of degranulation in MCs stimulated with anti-IgE (Figure 2, I-K), we defined the appearance of the first exteriorized granule structures as the beginning of $\mathrm{MC}$ degranulation in that cell, rather than the time at which the activation stimulus was first applied. We found no statistically significant difference in the rate of exteriorization of the total volume of $\mathrm{Av} . \mathrm{SRho}^{+}$granule structures whether the MCs were stimulated by anti-IgE or SP (Figure 5B). However, we observed that SP-mediated activation triggered the release of significantly higher numbers of Av.SRho ${ }^{+}$granule structures than did anti-IgE stimulation (Figure $5 \mathrm{C}$ ). By modeling the volumes of discrete budding Av.SRho ${ }^{+}$ granule structures during the first 60 seconds of degranulation, we found that anti-IgE stimulation resulted in the budding of Av.SRho ${ }^{+}$ secretory granule structures that exhibited a mean modeled volume of about $0.40 \mu \mathrm{m}^{3}$, many of which had heterogeneous shapes. By contrast, SP-mediated PBCMC activation triggered the exteriorization of substantially smaller and more spherical Av.SRho ${ }^{+}$ granule structures with a mean modeled volume of about $0.15 \mu \mathrm{m}^{3}$ (Figure 5, A and D). As depicted in Supplemental Figure 9, B and C, our TEM studies confirmed the Av.SRho fluorescence modeling results in showing that anti-IgE stimulation resulted in the exteriorization of a population of granule structures that contained many more large structures than did SP stimulation.

To extend our analysis of the physical characteristics of released granule structures no longer attached to the MC surface, we developed a 3D degranulation assay in which human MCs are embedded in a surrogate extracellular matrix gel. After 30 minutes of stimulation, we virtually isolated gel-trapped single Av.SRho granule structures released beyond the periphery of the cell from their still surface-bound counterparts and performed a computational 3D analysis to measure their size and sphericity (Supplemental Video 7). This showed that, compared with activation of PBCMCs with SP, anti-IgE-mediated activation of PBCMCs resulted in the release into the surrounding microenvironment of larger and more heterogeneously shaped granule structures (Figure 5, E-G).

These results indicate that the distinct intracellular secretory strategies triggered by SP versus anti-IgE activation (Figures 2-4) also result in the exteriorization of populations of MC secretory granule structures with different physical characteristics.
Differential regulation of the physical characteristics of mouse dermal MC granules during IgE-dependent or SP-induced MC activation in vivo. To analyze the features of MC granule secretion in vivo, we improved our detection method to enable 3D intravital imaging and modeling in the ear pinnae of anesthetized mice. We used passive cutaneous anaphylaxis (19) to study tissue MC activation by an IgE-dependent mechanism in vivo. Av.SRho was infused into the ear pinna dermis by intradermal (i.d.) injection 10 minutes before i.p. challenge, and Av.SRho fluorescence signal was assessed in living mice by 2-photon microscopy 30 minutes after i.p. challenge with specific antigen or vehicle (Figure 6A).

We investigated the presence of Av.SRho cells in $\mathrm{Mcpt5}-\mathrm{Cr} e^{+}$ $R 26 Y^{+}$mice, in which the Cre recombinase is under the control of the MC protease 5 (Mcpt5) promoter (which is reported to be specific for connective tissue-type and peritoneal MCs; ref. 55) to enable MC-selective expression of the enhanced yellow fluorescent protein (Mcpt5-EYFP) (56). When anti-DNP IgE-sensitized mice were injected with vehicle, no Av.SRho ${ }^{+}$cells were detected. However, after injection of DNP-HSA, we detected Av.SRho ${ }^{+}$ structures only surrounding $\mathrm{EYFP}^{+}$dermal MCs (Figure 6B). These results indicated that i.d. injection of Av.SRho is a useful new method to analyze MC degranulation in living animals by 2-photon microscopy.

We then performed a computational 3D analysis of individual exteriorized MC secretory granule structures 30 minutes after the induction of IgE-dependent or SP-dependent MC degranulation in the dermis of anesthetized Mcpt5-EYFP mice. After IgE-dependent local activation, tissue MCs exteriorized mainly large and nonspherical cytoplasmic granule structures (of high volumes and with a moderate modeled sphericity index), suggesting the occurrence of granule-granule fusion events associated with compound exocytosis in vivo. However, MCs activated by SP released mainly smaller and more spherical cytoplasmic granule structures, suggesting that they exteriorized a larger proportion of nonfused individual secretory granules (Figure 6, C-F, and Supplemental Video 8). These results show that mouse dermal MCs in vivo, like human MCs in vitro, can mobilize distinct patterns of degranulation in response to IgE-dependent versus SP-dependent activation.

MC-mediated cutaneous inflammation and systemic responses can exhibit different features depending on the nature of the stimulus. We next investigated whether the different patterns of MC granule secretion induced by SP- versus IgE-dependent mechanisms were associated with differences in the features of the ensuing MC-dependent vascular responses, which are thought to be highly dependent on the activities of mediators prestored in the cytoplasmic granules (57-60). We found that injection of $1 \mathrm{nmol}$ of SP or $5 \mathrm{ng}$ of DNP-HSA into anti-DNP IgE-sensitized $M c p t 5-\mathrm{Cre}^{+} \mathrm{R} 26 \mathrm{Y}^{+}$mice triggered a very similar percentage and intensity of MC degranulation, as measured by detection of Av.SRho ${ }^{+}$versus Av.SRho ${ }^{-} \mathrm{EYFP}^{+} \mathrm{MCs}$ in living mice by 2-photon microscopy 30 minutes after challenge (Supplemental Figure 12, A-C). We also confirmed these observations by quantifying MC degranulation in toluidine blue-stained sections of the challenged ear pinnae (Supplemental Figure 12D).

To quantify the enhanced vascular permeability induced by MC activation, we monitored Evans blue dye extravasation during the first 60 minutes following local injection of SP, DNP-HSA, or vehicle. In accord with our measurements of MC degranulation 
A
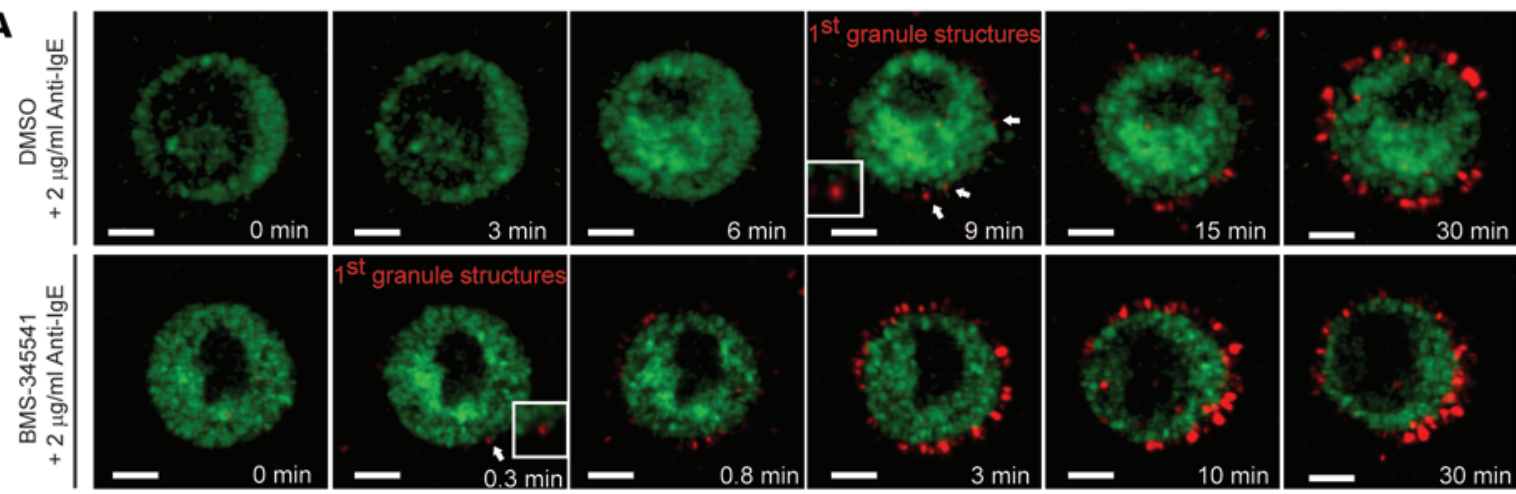

B

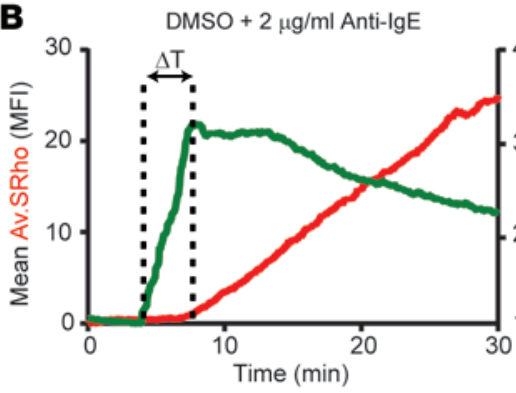

E

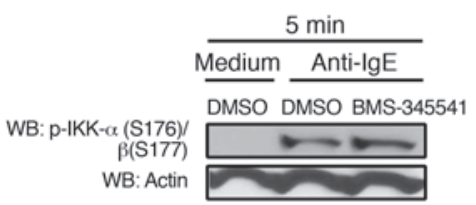

$\mathbf{F}$

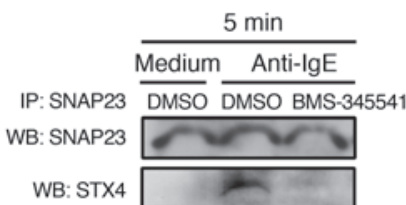

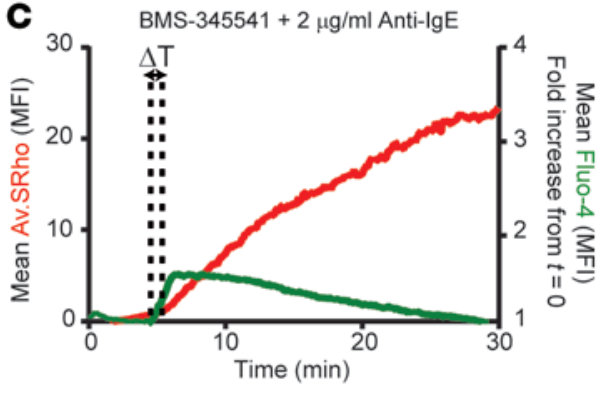

G

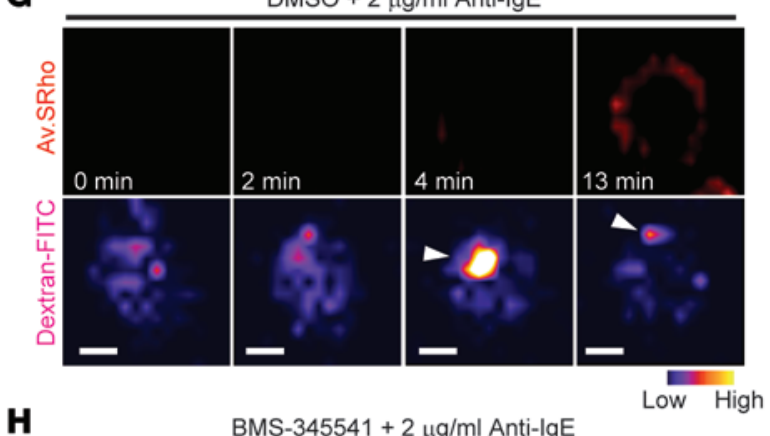

H

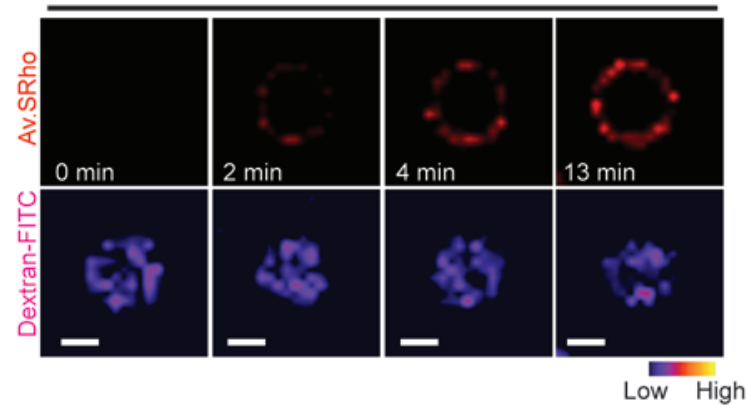

D

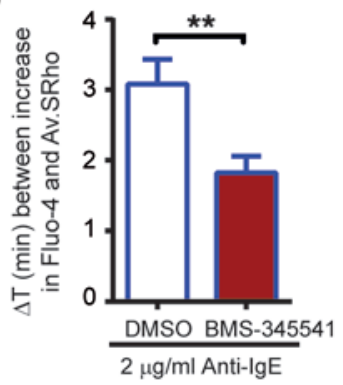

- DMSO $+2 \mu \mathrm{g} / \mathrm{ml}$ Anti-lgE

- BMS-345541 + $2 \mu \mathrm{g} / \mathrm{ml}$ Anti-lgE

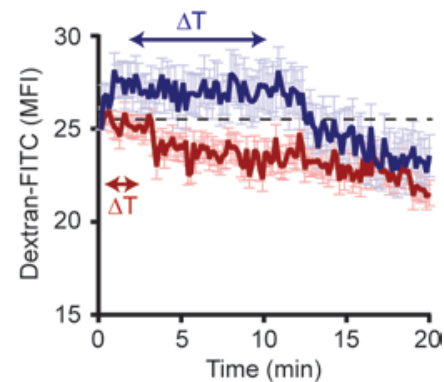

J

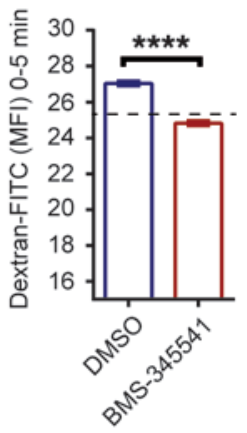

$\mathbf{K}$

DMSO + Medium

DMSO $+2 \mu \mathrm{g} / \mathrm{ml}$ Anti-IgE

BMS-345541 $+2 \mu \mathrm{g} / \mathrm{ml}$ Anti-lgE
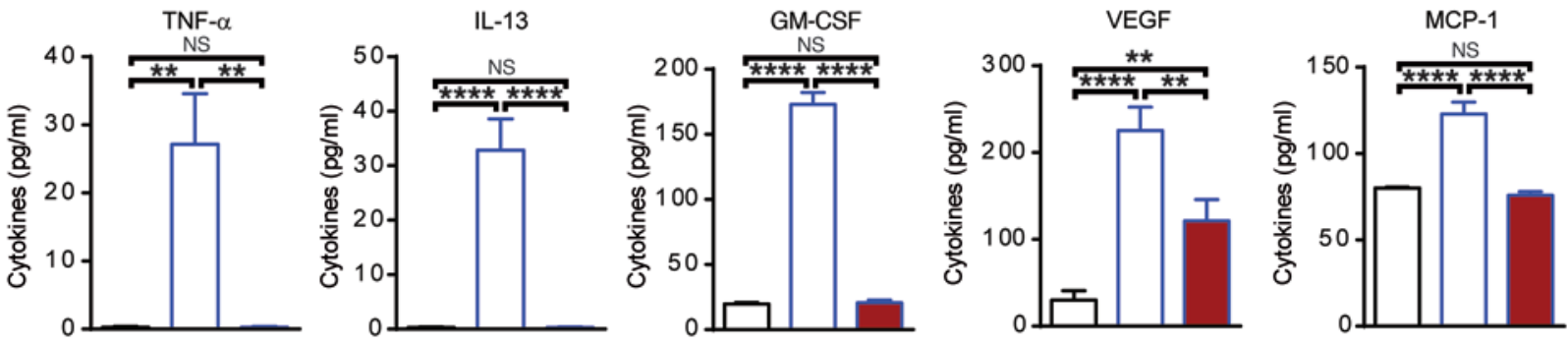
Figure 4. Pharmacological inhibition of IKK- $\beta$ activity changes the signaling and degranulation pattern of anti-IgE-activated MCs. (A-D) IgE-sensitized PBCMCs were pretreated for 60 minutes with $100 \mu \mathrm{M}$ BMS-345541 or DMSO and treated as described in Figure 2. (A) Upper panel: a PBCMC pretreated with DMSO; lower panel: a PBCMC pretreated with BMS-345541. White insets: higher magnification; arrows: granule structures; time scale reflects the kinetics of the responses. (B and C) Mean curves of Fluo-4 and Av.SRho MFI in PBCMCs pretreated with DMSO (B) or BMS-345541 (C). Dotted lines and arrows, $\triangle T$. (D) Mean $\triangle T$ in PBCMCs pretreated with DMSO (blue) or BMS-345541 (red). (E and F) PBCMCs pretreated with BMS-345541 or DMSO were stimulated for 5 or 15 minutes with anti-lgE. (E) Expression of phospho-IKK- $\alpha / \beta$ in PBCMC lysate. Actin was used as loading control. (F) Immunoprecipitation of SNAP23/STX4 complexes; immunoprecipitated SNAP23 was resolved and probed for STX4. (G-J) PBCMCs were pretreated with BMS-345541 or DMSO and treated as described in Figure 3C. Time-lapse of Av.SRho (red) and dextran-FITC (pseudocolor) in PBCMCs pretreated with DMSO (C) or BMS-345541 (H); white arrowheads, increases in dextran-FITC fluorescence. (I) Mean curve of dextran-FITC MFI in PBCMCs pretreated with DMSO (blue) or BMS-345541 (red). (J) Average of dextran-FITC MFI between $t=0$ and $t=5$ minutes. (K) Production of TNF- $\alpha$, IL-13, GM-CSF, VEGF, and MCP- 1 in PBCMCs stimulated 90 minutes with vehicle (black) or anti-lgE. ns, not significant. Mean $\pm \mathrm{SEM}$; 2-tailed, unpaired $t$ test; ${ }^{* *} P<0.01$; ${ }^{* * *} P<0.0001$. Data in A-D and G-J are from 3 independent experiments performed with PBCMCs from 2 donors ( $\sim 30$ PBCMCs analyzed per condition); data in $\mathbf{E}, \mathbf{F}$, and $\mathbf{K}$ are from 3 independent experiments, each of them performed with PBCMCs from a different single donor, each of which gave similar results. Scale bars: $5 \mu \mathrm{m}$.

dynamics (Figure 2), i.d. injection of SP triggered a rapid response with nearly maximum Evans blue extravasation within 15 minutes (Figure 7A). By contrast, i.d. injection of DNP-HSA into these mice (which had received an injection of anti-DNP IgE 16 hours before) induced a slower and more gradually developing response that did not reach the levels achieved in the response to SP until 60 minutes (Figure 7A). When we performed the same experiment in MC-deficient $\mathrm{Kit}^{W-s h / W-s h}$ mice, there were no statistically significant differences in the minimal levels of vascular leakage induced by vehicle, SP, or anti-DNP IgE and DNP-HSA, in accord with our previous findings indicating that the rapid cutaneous responses to i.d. SP (20) or IgE/antigen (61) in mice are largely if not entirely MC-dependent phenomena (Figure 7B). We also analyzed Evans blue dye extravasation in $M r g p r b 2^{M U T}$ mice, in which MRGPRB2 is inactivated by mutation (32). Intradermal injection of DNP-HSA induced substantial leakage of dye that was similar in both IgE-sensitized Mrgprb2 ${ }^{M U T}$ mice and Mrgprb2 ${ }^{+/+}$WT littermate mice (Figure 7C). By contrast, i.d. injection of SP triggered strong extravasation of dye in $\mathrm{Mrgprb2}{ }^{+/+}$littermate control mice but substantially lower responses in Mrgprb2 ${ }^{M U T}$ mice (Figure 7C). These results support the conclusion that much of the extravasation dynamics observed upon i.d. challenge with SP reflect largely MRGPRB2-mediated MC activation in vivo, with the residual small effect perhaps representing SP-dependent activation of other cells at the site (e.g., vascular endothelial cells that express NK1R; ref. 62). We also found that other MRGPRB2 agonists triggered MC-dependent extravasation dynamics similar to those observed following SP injection (Supplemental Figure 13).

We next measured swelling of the ear pinna after i.d. injections of vehicle, SP, or DNP-HSA. Intradermal injection of vehicle induced a weak and transient response that resolved within about 60-90 minutes (Figure 7D). SP or DNP-HSA induced similar lev- els of ear swelling during the first 60 minutes, but the swelling induced by SP resolved more rapidly than that induced by $\operatorname{IgE} /$ antigen (Figure 7D). Histological analyses of ear sections 6 hours after i.d. injections revealed fewer leukocytes in the SP-injected ear pinnae than in those challenged with IgE and antigen (Figure $7 \mathrm{E})$. By quantifying live leukocytes recovered from ear pinnae of mice sacrificed 6 hours after i.d. injection, we found that both SP and DNP-HSA injection induced significant increases in the numbers of $\mathrm{CD}_{4} 5^{+}$cells versus those in vehicle-injected sites. However, DNP-HSA injection sites harbored about twice as many CD $45^{+}$ cells (after subtraction of the numbers in vehicle-injected sites) than did sites injected with SP, with a significantly higher number of neutrophils and monocytes (Figure 7F).

We next administered specific antigen or SP intraperitoneally to induce systemic MC activation and investigated 2 pathophysiological consequences of such IgE-dependent versus SP-dependent MC activation: efflux of the intravascular tracer 70-kDa dextran-FITC and drop in body temperature. We used 2-photon microscopy to quantify the efflux of dextran-FITC by measuring the changes in fluorescence intensity in the interstitial space (63) after induction of MC activation. When mice were injected with vehicle, minimal amounts of dextran-FITC extravasation were detected in the interstitial space (Supplemental Figure 14, A-C, and Supplemental Video 9). Induction of an IgE-dependent reaction was associated with a relatively slow but progressively increasing response, with the highest levels of dextran-FITC detected in the interstitial space about $30 \mathrm{~min}-$ utes after induction of the reaction. By contrast, SP triggered a much faster response that reached nearly maximum tracer leakage within 10-15 minutes (Supplemental Figure 14, A-C, and Supplemental Video 9).

Systemic responses to SP were characterized by a very fast and transient drop of body temperature (about $-2.5^{\circ} \mathrm{C}$ ), reaching the lowest values within 10-15 minutes of i.p. injection of SP (Supplemental Figure 12D). However, IgE-dependent passive systemic anaphylaxis was associated with a more progressive but substantially stronger (about $-5^{\circ} \mathrm{C}$ ) and more sustained drop in body temperature (Supplemental Figure 14D). In MC-deficient Kit ${ }^{W-s h} / W-s h$ mice, SP induced minimal responses (which, with the numbers of mice tested, were not statistically significant versus responses in vehicle-treated WT mice), perhaps reflecting actions of SP on neurokinin 1 receptor-bearing (NK1R-bearing) cells (not MCs), which can be activated by SP (62), and IgE and specific antigen induced no detectable responses (Supplemental Figure 14D).

These data demonstrate that when mice are challenged i.p. with SP or IgE/DNP-HSA, SP induces a rapid systemic response (associated with the rapid enhancement of vascular permeability in the skin and a rapid drop in body temperature that also rapidly resolves), whereas the corresponding responses to IgE and antigen are characterized by the slower enhancement of vascular permeability in the skin and a bigger and more sustained drop in body temperature. These features of the systemic responses to SP- or IgE-dependent MC activation, like those of the cutaneous responses to local induction of SP- or IgE-dependent MC activation (Figure 7, A, B, and D), parallel the features we observed in the patterns of MC degranulation induced by SP- versus IgEdependent activation in vitro. 
A Pictures taken $40 \mathrm{sec}$ after detection of first granule structures
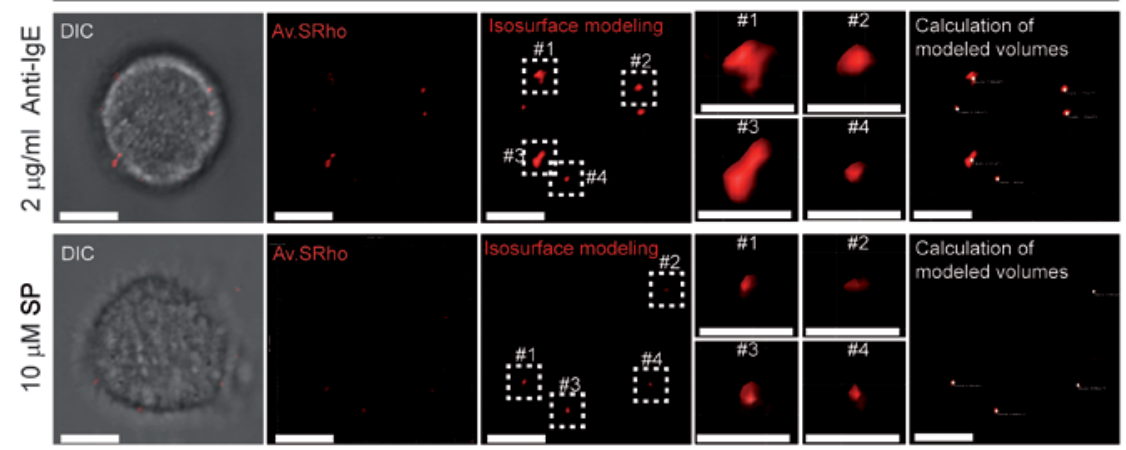

B

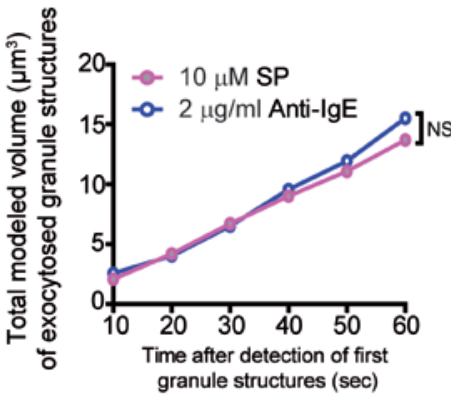

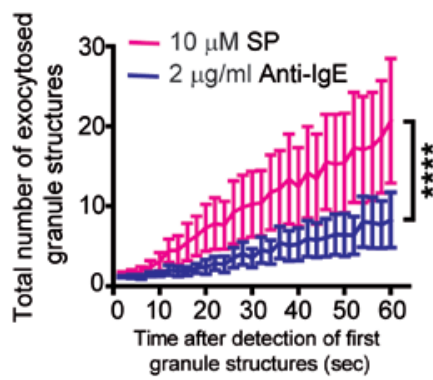

D

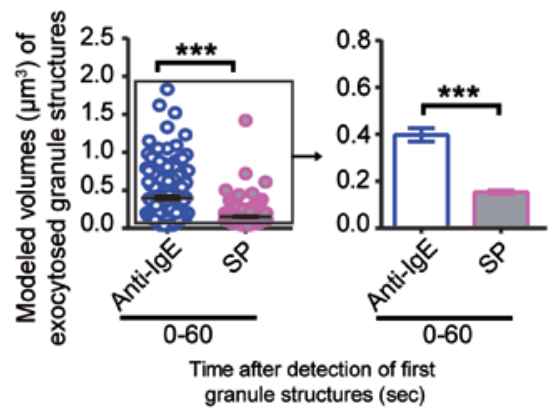

$\mathbf{E}$

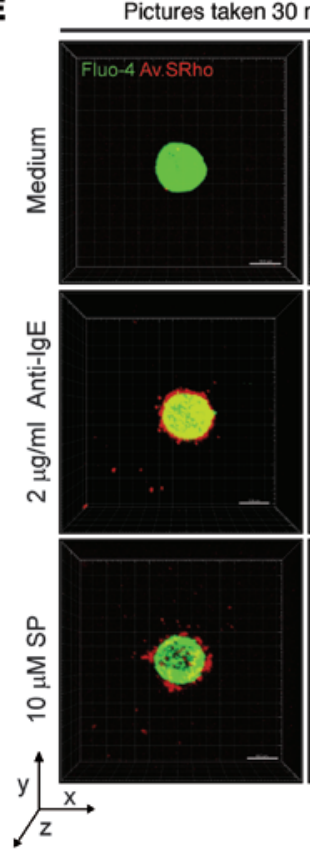

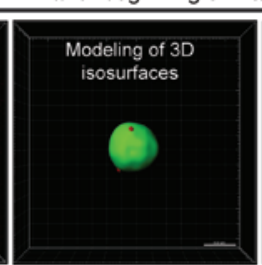
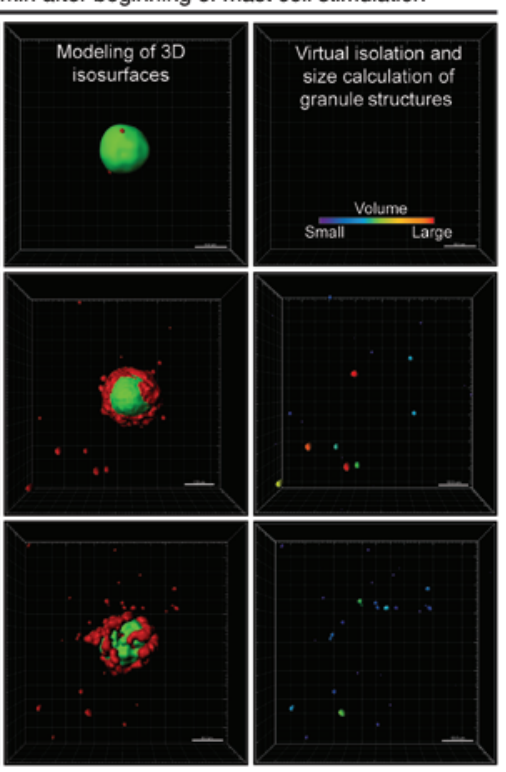

$\mathbf{F}$

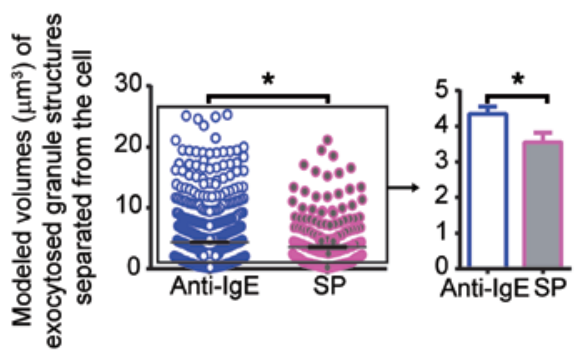

G

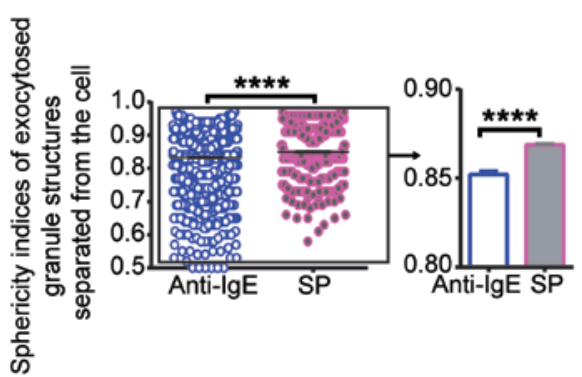

Figure 5. The features of exteriorized human MC granule structures can vary depending on the stimulus. (A) Photographs of PBCMCs activated with anti-IgE (upper panels) or SP (lower panels). From left to right: Av.SRho (red) merged with differential interference contrast (DIC; gray); Av.SRho (red); isosurface modeling (red) of Av.SRho; modeled granule structures; modeled volume calculation. (B) Total accumulated volume of budding granule structures (i.e., total amount of externalized Av.SRho+ granule volume per MC) in cubic micrometers. (C) Total numbers of exocytosed Av.SRho ${ }^{+}$granule structures. (D) Modeled volumes of budding granule structures (in cubic micrometers, assessing the size of granule structures still attached to the MC surface) per single MC during the first minute after the start of degranulation, following anti-lgE (blue) or SP (pink) stimulation. (E) 3D photographs of single PBCMCs embedded in a matrix gel, 30 minutes after exposure to medium (no stimulation, upper panels), antiIgE (middle panels), or SP (lower panels). Panels, left to right: Av.SRho (red) merged with Fluo-4 (green); isosurface modeling of Av.SRho (red) and Fluo-4 (green); virtual isolation of released granule structures (structures are pseudocolored based on their volume). (F) Modeled volumes (in cubic micrometers, assessing granule structures not attached to the MC surface). (G) Modeled sphericity indices (from 0 to 1,1 being a perfect sphere). Data shown in $\mathbf{F}$ and $\mathbf{G}$ were obtained 30 minutes after the beginning of mast cell stimulation. (B and $\mathbf{C})$ Mean \pm SEM; 2-way ANOVA. (D, F, and $\mathbf{G}$ ) Left panel: each dot represents 1 individual granule structure analyzed; right panel: mean \pm SEM of the data shown in the left panel. Data are from more than 200 granule structures analyzed per condition. Two-tailed, unpaired $t$ test; ${ }^{*} P<0.05 ;{ }^{* * *} P<0.001 ;{ }^{* * *} P<0.0001$. Data are from 3 independent experiments, each of them performed with PBCMCs from a different single donor, each of which gave similar results. Scale bars: $5 \mu$ m. 
Different effects on popliteal draining lymph nodes of footpad injections of IgE and antigen versus SP. Draining lymph nodes (DLNs) can respond to distal events by undergoing rapid enlargement. It recently has been reported that cytoplasmic granules released from activated MCs in the mouse footpad can deliver signals (e.g., TNF stored in the MC granule matrix) from the periphery to DLNs, and that this can contribute to the enlargement of such DLNs (25). We wondered whether the different physical characteristics of exteriorized MC granule structures following IgE-dependent versus SP-dependent stimulation in vivo might influence their capacity to reach and elicit enlargement of local DLNs.

First, we determined whether injections of $1 \mathrm{nmol}$ of SP into the right footpad and $5 \mathrm{ng}$ of DNP-HSA into the contralateral anti-DNP IgE-sensitized left footpad of the same mouse would cause degranulation of MCs resident in either the challenged footpads or the draining popliteal lymph nodes. These doses of the 2 stimuli were chosen because, when injected i.d., they induced a similar percentage and extent of dermal MC degranulation (Supplemental Figure 12). We found that these doses of SP and DNP-HSA also induced, by 2 hours after injection into the footpads, a similar extent of Evans blue extravasation (Figure $8 \mathrm{~A}$ ) and MC degranulation (Figure 8B), whereas the MCs resident in the DLNs exhibited a "nondegranulated" phenotype (Figure 8C). Two hours after footpad injection of DNP-HSA (but only rarely after injection of SP), we detected in the periphery of the DLNs small particles that stained metachromatically (in toluidine blue-stained sections) and that were Av.SRho+ (Figure 8D), observations consistent with those of Kunder et al. (25) indicating that MC granules secreted extracellularly can be transported to the local DLNs. When we repeated such experiments but assessed DLNs 24 hours after the footpad injections, we found that those LNs draining the IgE- and antigen-injected footpads had enlarged significantly (by $\sim 90 \%$ ) versus the DLNs of vehicle-injected footpads (Figure 8, E and F). By contrast, based on the numbers of mice tested, the difference in the approximately $28 \%$ higher mean weight of the lymph nodes draining the SPinjected versus the vehicle-injected footpads was not statistically significant (Figure 8F). These data suggest that the granule structures generated following IgE-dependent MC activation are more likely to be transported to and/or persist in the DLNs than those generated after SP-induced MC activation.

\section{Discussion}

It has long been recognized that MC degranulation can be induced both by antibody-dependent mechanisms that activate MCs via Fc receptors, especially FceRI (8), and also by diverse stimuli that function independently of antibodies and Fc receptors, many of them by activating MCs via GPCRs (17, 19, 20, $32,64)$. These 2 different forms of stimulation of various MC populations in vivo or generated in vitro also can induce such cells to synthesize and release newly formed lipid mediators and cytokines. However, the amounts of such nonstored mediators released by MCs can vary depending on the particular MC populations analyzed and the details of the conditions of stimulation (e.g., see refs. 32, 65-67). Therefore, we focused our in vitro and in vivo analysis mainly on the early stages of MC activation, the period during which mediators stored in the MC's cytoplasmic granules are thought to have important roles in MC-dependent inflammatory responses $(16,60)$.

Here we provide in vitro and in vivo evidence demonstrating that MCs can respond to FcR-dependent signals and signals transmitted via a variety of GPCRs by mobilizing distinct spatiotemporal patterns of exteriorization of their cytoplasmic granules. A similar pattern of granule exteriorization was observed in human MCs stimulated by either of 2 FcR-dependent signals, IgE/anti-IgE or IgG immune complexes. This pattern is characterized by a 3- to 5 -minute time partition between the onset of intracellular signaling, as assessed by the first changes in intracellular calcium flux, and the onset of a relatively prolonged period of exteriorization of Av.SRho ${ }^{+}$granule matrices, which is preceded by evidence of the deacidification of cytoplasmic granules, most likely indicating that some (perhaps transient) fusion of granule membranes with the plasma membrane occurs even before Av.SRho ${ }^{+}$granule structures are detectable at the cell surface. By contrast, in response to stimulation by SP, C3a, C5a, or ET1, MCs very quickly reach a peak of intracellular calcium that is rapidly followed by the fast secretion of structures that, based on their size and sphericity features, likely represent individual secretory granules. A hypothetical model based on our findings is presented in Figure 9.

Our evidence indicates that IKK- $\beta$ has an important role in determining whether PBCMCs exhibit an "IgE-dependent" or "SP-induced" spatiotemporal pattern of degranulation. Notably, the role of IKK- $\beta$ (also known as IKK2) in MC degranulation has been investigated by 2 groups that have come to different conclusions. Using fetal liver-derived MCs (FLMCs) from WT versus $I K K 2^{-/-}$mice and employing engraftment of MC-deficient $K i t^{W / W-v}$ mice with such FLMCs, Suzuki et al. (47) reported that IKK- $\beta$ was required for optimal IgE-dependent $\beta$-hexosaminidase release, SNAP23/STX4 complex formation, and IgE-dependent anaphylaxis reactions in vivo. However, Peschke et al. (48) recently demonstrated, using Mcpt5-Cre $e^{+} I K K 2^{f / f l}$ versus $M c p t 5-C r e^{-} I K K 2^{f / f l}$ littermate mice, and peritoneal cell-derived MCs derived from such mice, that MC-specific deletion of IKK- $\beta$, which in their mice occurred after the MC lineage had sufficient expression of Mcpt5 to delete $I K K 2$, did not significantly influence IgE-dependent $\beta$-hexosaminidase release at 30 minutes, SNAP23 phosphorylation in vitro, or the intensity of IgE-dependent systemic or cutaneous passive anaphylaxis reactions in vivo.

In our studies of primary human MCs derived from peripheral blood progenitors of healthy donors (PBCMCs), we found that IKK- $\beta$ activity was not required for MC degranulation induced by either anti-IgE (in accord with the findings of Peschke at al.) or SP. In accord with the findings of Suzuki et al., our pharmacological data indicate that IKK- $\beta$ activity importantly controls the SNAP23/ STX4-dependent process that enables MCs to orchestrate the spatiotemporal pattern of degranulation exhibited by cells stimulated by anti-IgE. However, because similar levels of secretion of the granule-stored mediator $\beta$-hexosaminidase can be achieved at 30 minutes after stimulation of human PBCMCs by either SP or anti-IgE (e.g., see Figure 1A), it is possible that inhibition of IKK- $\beta$ and the subsequent change from an "anti-IgE-like" to an "SP-like" pattern of MC degranulation might have little or no effect on the total amount of degranulation and $\beta$-hexosaminidase released in response to the 2 different stimuli. 
A Intravital analysis of physical characteristics of mast cell granule structures

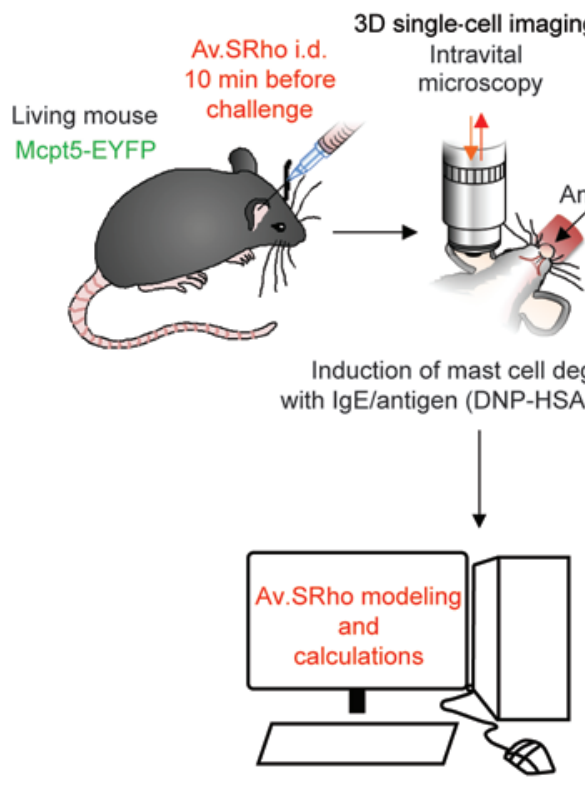

B

Pictures taken $\mathbf{3 0}$ min after injection of vehicle or DNP-HSA
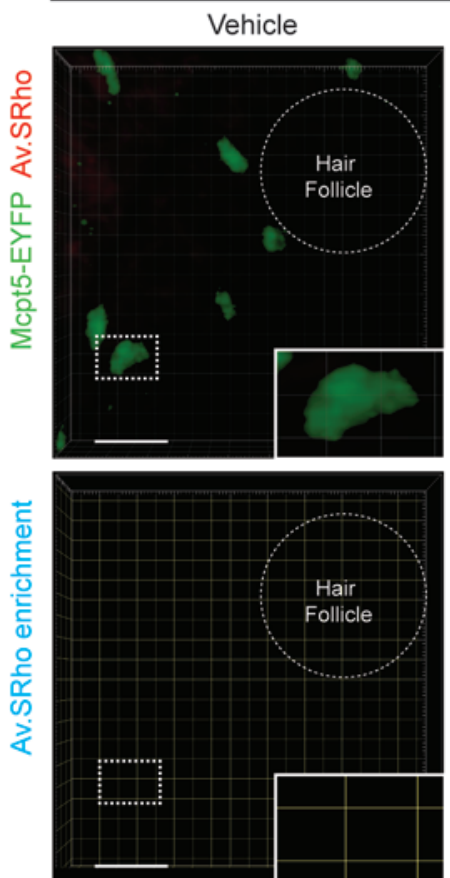

Low High

C

Pictures taken 30 min after injection of vehicle, DNP-HSA or SP
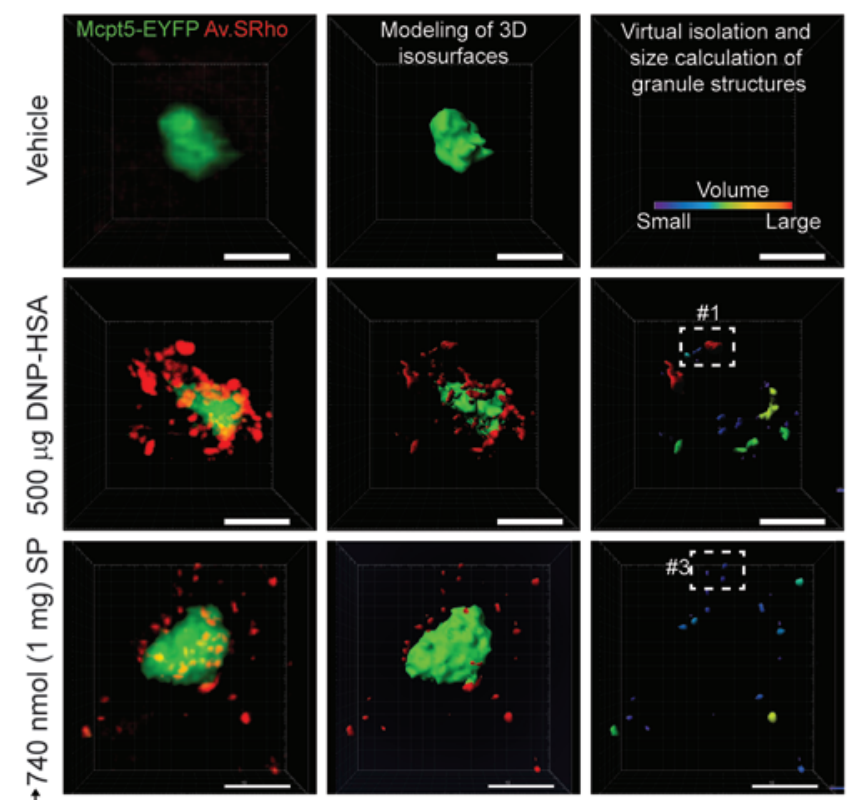

$y \underset{z}{\underset{z}{x}}$

E
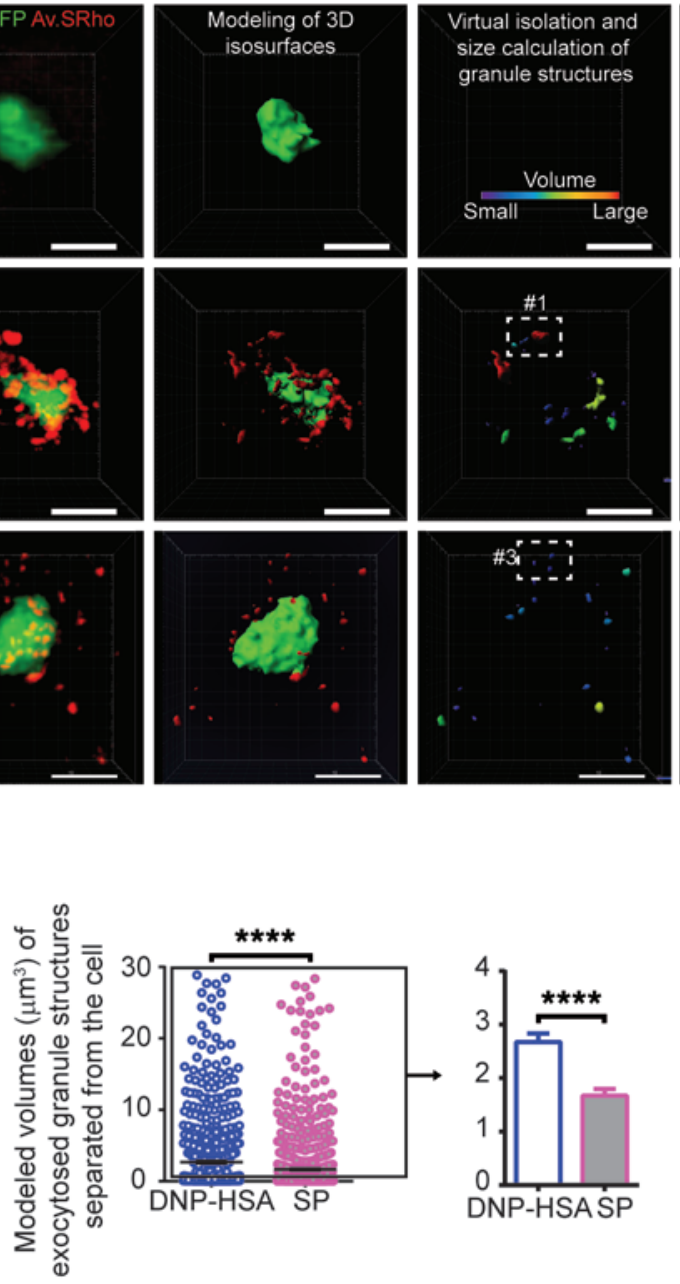
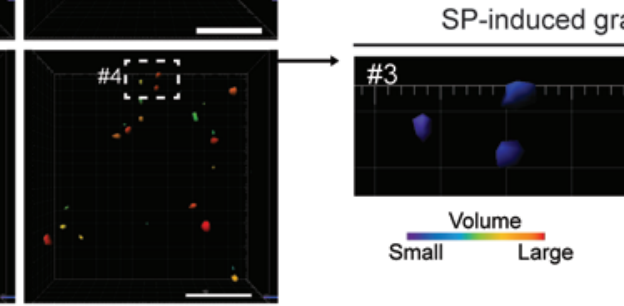

$\frac{\text { Volume }}{\text { Small Large }}$

$\mathbf{F}$

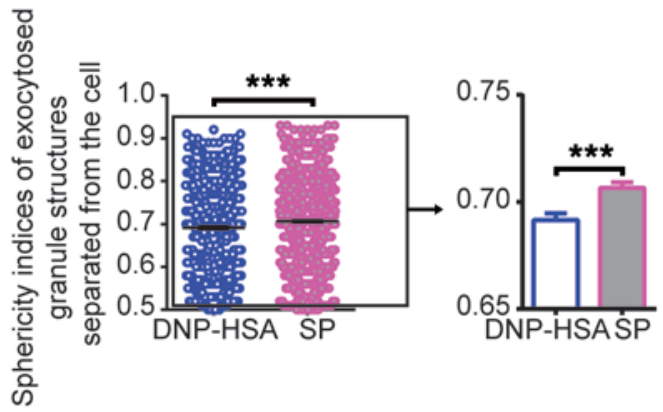


Figure 6. Differences in the features of granule structures exteriorized after Fc\&RI- or MRGPRB2-mediated activation of mouse MCs in vivo. (A) Mcpt5-Cre+ R26Y+ (Mcpt5-EYFP) mice were sensitized or not by i.d. injection into the ear pinna of 20 ng of mouse anti-2,4-dinitrophenyl (anti-DNP) IgE. Sixteen hours later, $8 \mu \mathrm{g}$ of Av.SRho was injected i.d. into the same ear pinna. Nonsensitized mice were injected i.p. with $1 \mathrm{mg}$ of SP IgE-sensitized mice were injected i.p. with $500 \mu \mathrm{g}$ of DNP-HSA. In control experiments, sensitized mice were injected i.p. with vehicle. 3D high-resolution single-cell images were taken, and Av.SRho fluorescence signal was modeled. (B) 3D photographs of the ear pinna 30 minutes after injection of vehicle (left panels) or DNP-HSA (right panels). Upper panels: Av.SRho (red) and EYFP (green) merged; lower panels: Av.SRho shown in pseudocolor intensity. Scale bars: $20 \mu \mathrm{m}$. (C) 3D analyses of ear pinna MC granule structures, 30 minutes after injection of vehicle (upper panels), DNP-HSA (middle panels), or SP (lower panels). Panels, left to right: Av.SRho (red) and EYFP (green) merged; isosurface modeling of Av.SRho (red) and EYFP (green); virtual isolation of released granule structures. Scale bars: $10 \mu \mathrm{m}$. (D) 3D modeled granule structures stained with pseudocolor as a function of their volume or their sphericity index. (E) Modeled volumes of granule structures (in cubic micrometers) following anti-lgE (blue) or SP (pink) injection. (F) Modeled sphericity indices of granule structures, following anti-IgE (blue) or SP (pink) injection. Left panel: each dot represents 1 individual granule structure analyzed; right panel: mean \pm SEM of the data represented in the left panel. Two-tailed, unpaired $t$ test; ${ }^{* *} P<0.001$; ${ }^{* * * *} P<0.0001$. Data represent approximately 1,000 single-granule structures analyzed from 3 experiments performed with 3 mice per condition, each of which gave similar results.

It should be noted that the Suzuki et al. study (47) and the Peschke et al. study (48) (using 2 different types of mouse MCs) and our study of human PBCMCs each analyzed distinct MC populations, and that this may have contributed to some of the differences in the results of these studies. Nevertheless, our study suggests that, in human PBCMCs, instead of controlling the "quantity" of MC granule secretion, IKK- $\beta$ and SNAP23 may be more important in regulating the "quality" of the response to FceRI-dependent activation by helping to orchestrate the "antiIgE-like" compound exocytosis degranulation strategy. Finally, while our studies of the spatiotemporal characteristics of MC degranulation were performed with 2 types of FcR-dependent MC activation and in MCs stimulated with 7 different agonists acting via 4 distinct GPCRs, we have analyzed the roles of IKK- $\beta$ and SNAP23 only in human PBCMCs activated by anti-IgE and SP. Additional work would be required to assess whether the same findings would be obtained with other stimuli of MC activation, or in other populations of MCs. Indeed, based on TEM studies, it has been suggested that the extent to which anti-IgE stimulation can induce evidence of granule-granule fusion prior to exteriorization of granule structures that is detectable by TEM may be greater in the case of human lung MCs (38) than of human skin MCs (39), raising the possibility that different $\mathrm{MC}$ populations in a single species may exhibit variation in the spatiotemporal features of degranulation induced by the same stimulus.

There is also evidence that, depending on the nature of the stimulus, the same receptor can induce different functional responses in the same population of MCs. Notably, the group of Juan Rivera recently reported that FceRI can discriminate high-affinity stimulation from low-affinity stimulation, which elicit different effector responses (68). They analyzed FceRI phosphorylation, cluster size, mobility, and distribution, as well as cytokine/chemokine secretion, when mouse bone marrow-derived cultured MCs (BMCMCs) were stimulated with 2 carefully chosen high- versus low-affinity antigens that induced about $50 \%$ versus less than $20 \%$ degranulation, respectively. Suzuki et al. (68) also analyzed the shape and the intensity of changes in intracellular calcium levels when BMCMCs were stimulated with the high- versus low-affinity antigens. Interestingly, while the intensity of the calcium signal was greater with the high-affinity antigen than with the low-affinity antigen (consistent with the observation of more degranulation in response to the high-affinity antigen), the shape of the calcium signal was very comparable for the 2 stimulations: sustained for a prolonged period of time (see Figure 3C in Suzuki et al., ref. 68). These observations align with our findings, in that we showed a very similar pattern of sustained intracellular calcium levels when human MCs were activated with either of 2 different stimuli that operated through FcRs (i.e., anti-IgE and IgG immune complexes), and, in the case of anti-IgE, regardless of the strength of stimulation (Figure 2I and Supplemental Figure $6 \mathrm{H})$. Although the Suzuki et al. study (68) and the present study investigated different aspects of MC biology, we think that the 2 studies provide complementary information about distinct mechanisms that can influence the production of inflammatory mediators by MCs and thereby regulate their effector functions.

We also think that the existence of spatiotemporally distinct MC degranulation patterns that can be elicited in response to different types of stimuli acting via different receptors has implications for our understanding of $\mathrm{MC}$ functions in vivo. We found that activation of skin MCs by SP in vivo resulted in rapidly developing vascular reactions, whereas IgE-dependent reactions took longer to reach the same intensity of dye extravasation. We also showed that while SP induced brief local inflammatory reactions, IgE-dependent reactions were generally more intense and lasted for a more prolonged period of time. Similar results were obtained when systemic responses to IgE and antigen or SP were compared. Given the importance of the preformed mediators stored in MC granules for the early stages of MC-dependent inflammatory responses (57-60), we hypothesize that the differences in the features of SP-dependent versus IgE-dependent responses in vivo reflect, at least in part, the differences in the spatiotemporal features of MC degranulation in these 2 settings, as well as possible differences in the de novo synthesized mediators that MCs stimulated via IgE-dependent versus SP-dependent mechanisms produce in these reactions.

Our findings also offer new ways to think about old clinical observations. For example, it has been recognized clinically that the features of pseudoallergic reactions (18), such as those induced by injection into the skin of drugs now known to activate MCs (69) by binding to MRGPRX2 (32), can differ from those induced by the injection of allergens into the skin of sensitized subjects (which results in the IgE-dependent activation of cutaneous MCs at that site). Pseudoallergic skin reactions are often transient (e.g., ref. 69), whereas IgE-dependent cutaneous reactions can be longer-lasting and result in more persistent local inflammation (e.g., ref. 70). The persistence of inflammation at sites of IgE-dependent MC activation generally has been attributed to the effects of de novo synthesized MC mediators, such as lipid mediators and cytokines $(7,70)$. Our findings indicate that IgE-depen- 
A Evans blue extravasation $60 \mathrm{~min}$

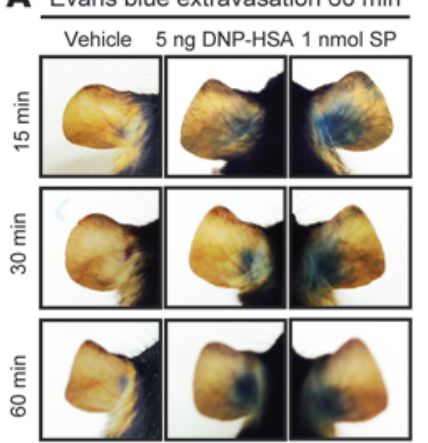

$-\infty$ Vehicle i.d. $(n=6)$
-05 ng DNP-HSA i.d. $(n=6)$
$-\infty 1$ nmol SP i.d. $(n=6)$

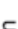

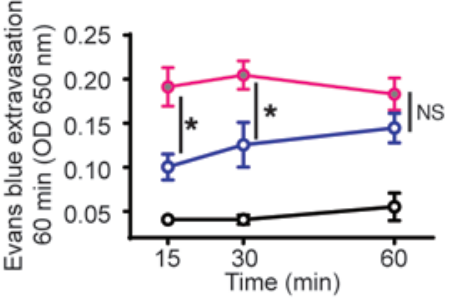

$\square$ Vehicle i.d. $(n=6)$

$\square$ ng DNP-HSA i.d. $(n=6)$

$1 \mathrm{nmol}$ SP i.d. $(n=6)$

C
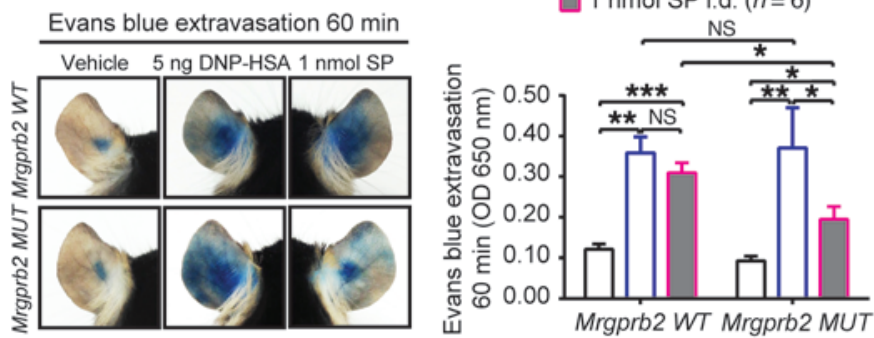

B

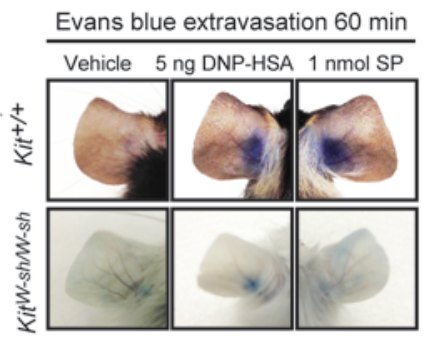

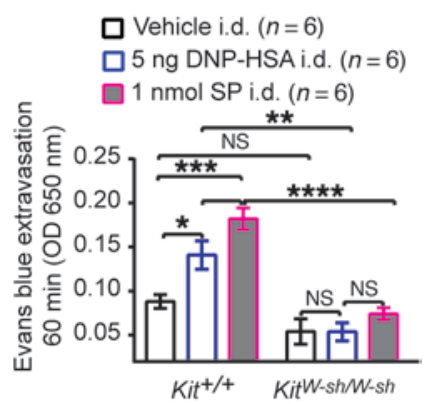

d. $(n=6)$

SP i.d. $(n=6)$
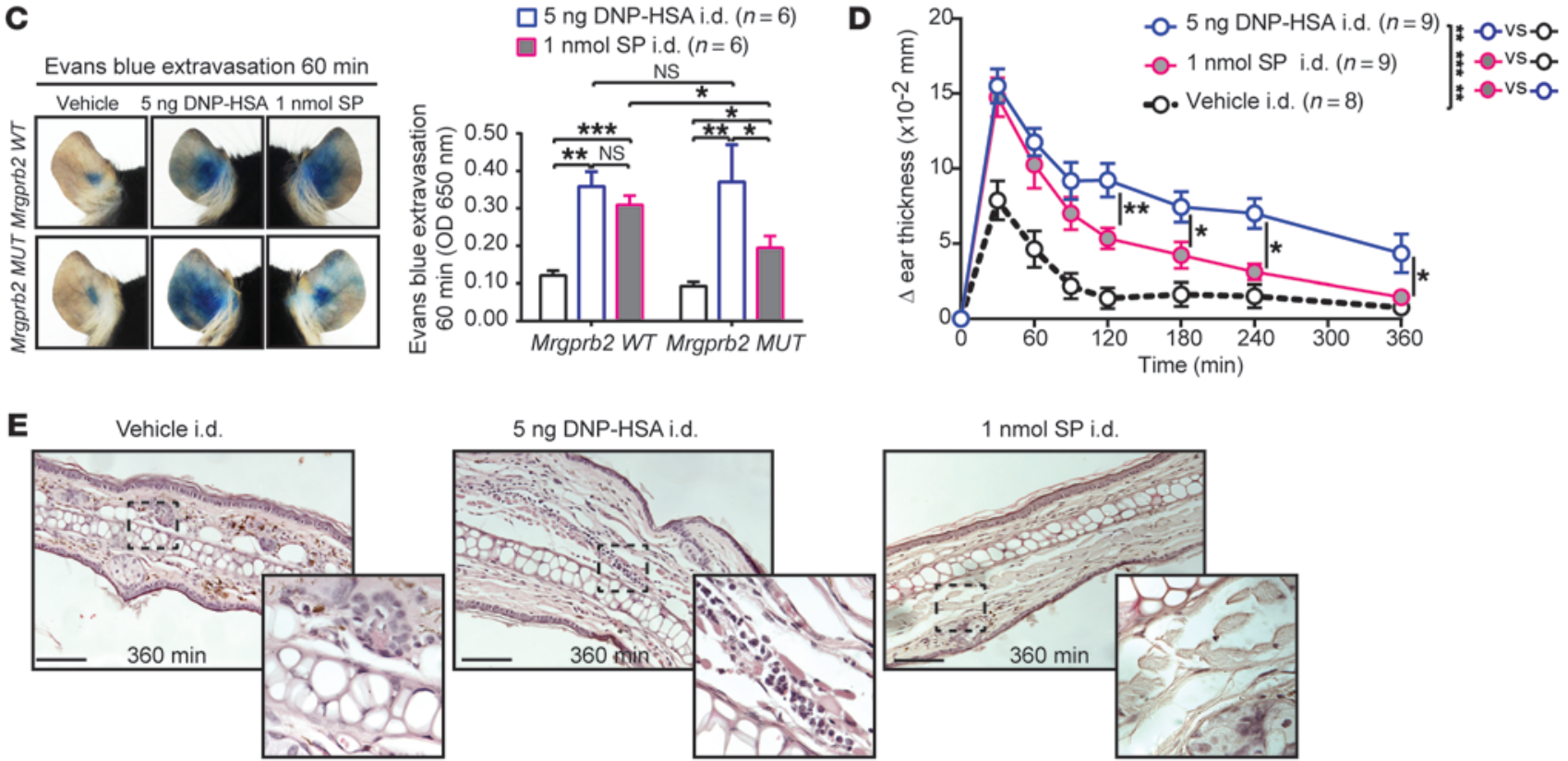

$\mathbf{F}$
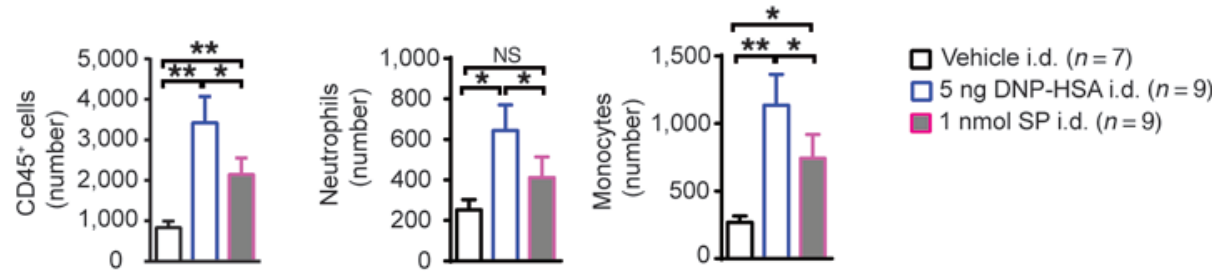

$\square$ ng DNP-HSA i.d. $(n=$

1 nmol SP i.d. $(n=9)$

Figure 7. MC-mediated cutaneous inflammation exhibits different features depending on the nature of the MC activation stimulus. Mice were sensitized by i.d. injection of $20 \mathrm{ng}$ anti-DNP IgE into the left ear pinna, and vehicle alone was injected i.d. into the right ear pinna. Sixteen hours later, the right ear pinna was injected i.d. with $1 \mathrm{nmol}$ of SP (pink) and the left with $5 \mathrm{ng}$ of DNP-HSA (blue). In control experiments, both ear pinnae were injected i.d. with vehicle (black). (A) Evans blue extravasation in the ears of C57BL/6 WT mice. Left panel: representative photographs; right panel: quantification of Evans blue extravasation (OD $650 \mathrm{~nm}$ ). (B) Same experiment as in A, but performed in MC-deficient C57BL/6 Kit ${ }^{\text {W-sh/W-sh }}$ mice versus C57BL/6 WT mice. (C) Same experiment as in A, but performed in Mrgprb2 ${ }^{M U T}$ mice versus littermate WT mice. (D) Changes $(\Delta)$ in ear thickness over time after i.d. injection of WT mice. (E) Representative H\&E photomicrographs of sections of ear pinnae in mice sacrificed 360 minutes after i.d. injection. Scale bars: $200 \mu$ m; insets show boxed areas at higher magnification $(\times 40)$. (F) Flow cytometry analysis of cells (CD45+ cells, neutrophils and monocytes) recovered from ear pinnae 360 minutes after i.d. injection of C57BL/6 mice. (A-D and F) Mean \pm SEM; 2-tailed, unpaired $t$ test (vehicle vs. SP or vehicle vs. DNP) or paired $t$ test (SP vs. DNP-HSA). Two-way ANOVA (SP vs. DNP-HSA, DNP-HSA vs. vehicle and SP vs. vehicle); ${ }^{*} P<0.05 ;{ }^{* *} P<0.01 ;{ }^{* *} P<0.001 ;{ }^{* * * *} P<0.0001$. Data were pooled from 3 independent experiments, each of which gave similar results. $n=$ total number of mice per condition.

dent and MRGPRX2-dependent MC activation elicit patterns of MC degranulation that differ in spatiotemporal features and in the physical characteristics of the released granule structures. These differences, as well as differences in the de novo synthesized mediators released by MCs at such sites, may contribute to differences in the clinical features of such reactions.
Finally, we provide evidence that the (on average) larger and less spherical granule structures generated following IgEdependent reactions elicited in the mouse footpad, as compared with the (on average) smaller and more spherical granule structures released upon SP stimulation, were more likely to travel to and/or persist in DLNs (25), a finding that was associated with 
A

Evans blue extravasation 2 hours after footpad injection

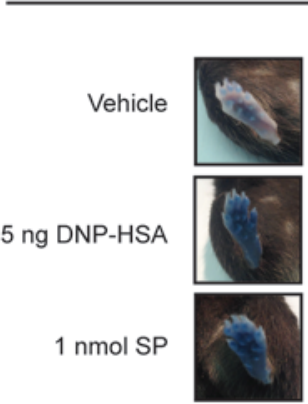

Vehicle i.d. $(n=3)$

$\square$ ng DNP-HSA i.d. $(n=5)$

1 nmol SP i.d. $(n=5)$

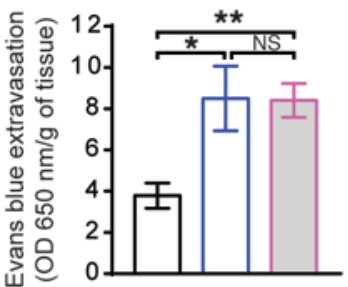

B

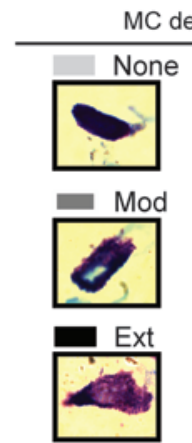

MC degranulation 2 hours after footpad injection

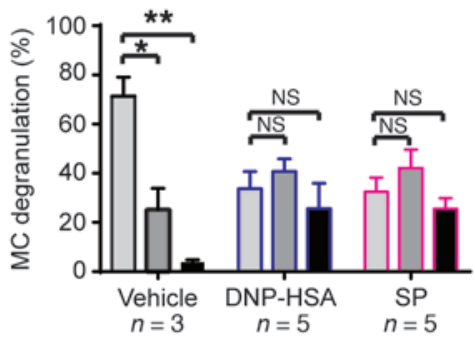

C

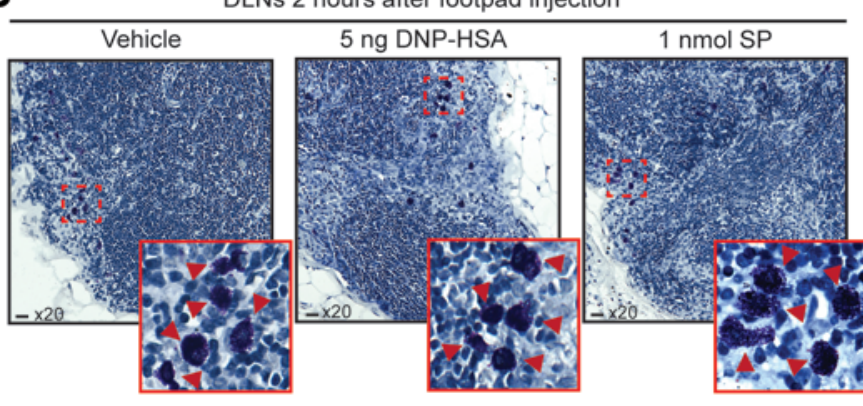

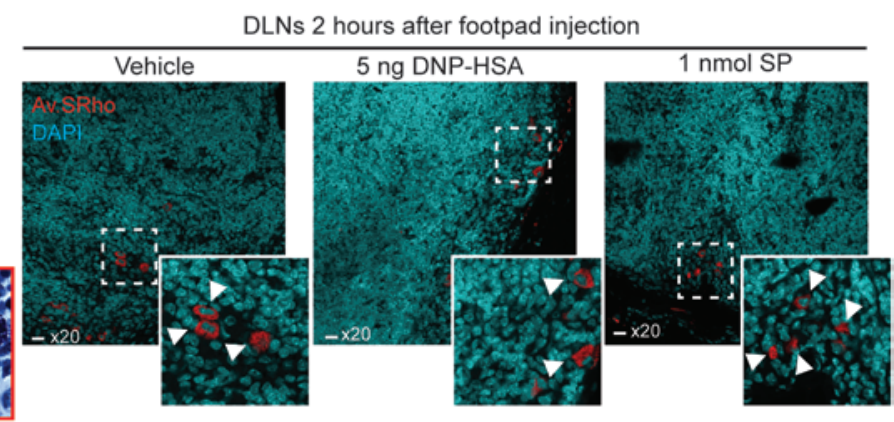

D DLNs 2 hours after footpad injection

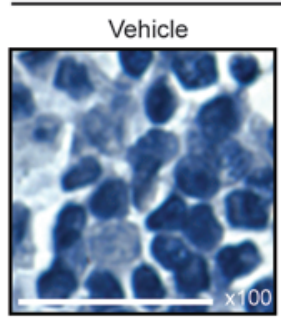
5 ng DNP-HSA $1 \mathrm{nmol} \mathrm{SP}$
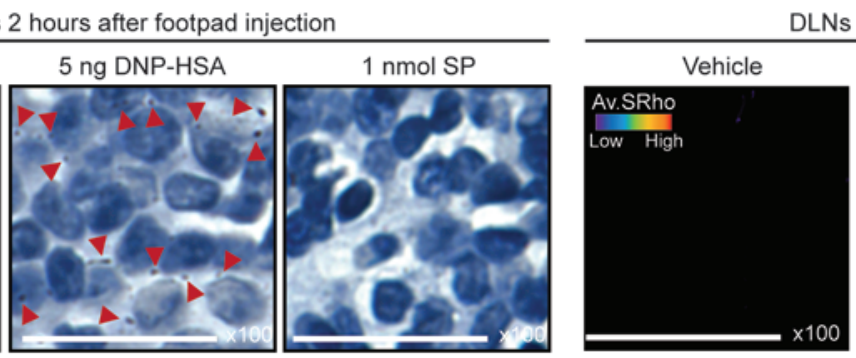

$\mathbf{E}$

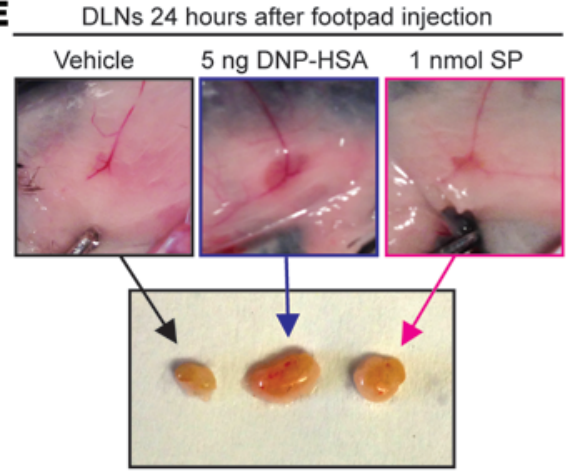

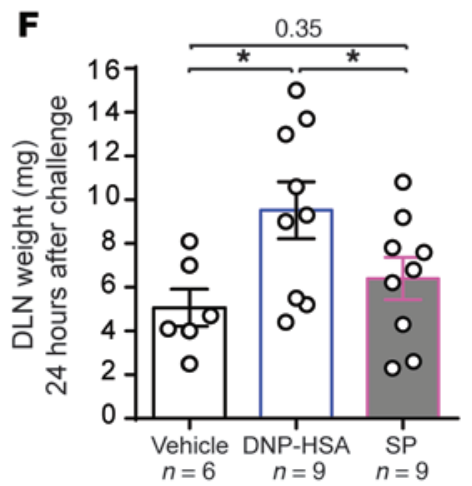

Figure 8. Greater enlargement of DLNs is induced by localized MC activation by IgE and antigen than by SP. Mice were locally sensitized by injection of $20 \mathrm{ng}$ anti-DNP IgE into the left footpad, and vehicle alone was injected into the right footpad. Sixteen hours later, the left footpad was injected with 5 ng DNP-HSA (blue) and the right footpad with $1 \mathrm{nmol}$ SP (pink). In control experiments, both footpads were injected with vehicle (black). (A) Evans blue extravasation in the footpad of WT mice 2 hours after injection. Left panel: photographs; right panel: quantification of extravasation (OD $650 \mathrm{~nm}$ per gram of tissue). (B) Quantification of MC degranulation in the footpad 2 hours after injection; MC degranulation determined by classification in 3 categories: not degranulated (None, gray), moderately degranulated (Mod, darker gray), and extensively degranulated (Ext, black). (C) Micrograph of toluidine bluestained sections (left panel) and Av.SRho (red) merged with DAPI (cyan) (right panel) of DLNs 2 hours after footpad injection. Red and white (left and right panel, respectively) insets show boxed areas at higher magnification (×60). Red (left) or white (right) arrowheads in insets depict nondegranulated MCs. (D) Visualization of MC granules in toluidine blue-stained sections (left panel) and Av.SRho-stained sections (pseudocolor enrichment, right panel) of DLNs 2 hours after footpad injection. Scale bars: $50 \mu \mathrm{m}$. (E) Photographs showing appearance of DLNs in situ (upper panel) and ex vivo (lower panel) 24 hours after footpad injection. (F) Weight of DLNs 24 hours after footpad injection. Mean \pm SEM; 2-tailed, unpaired $t$ test (vehicle vs. SP or vehicle vs. DNP) or paired $t$ test (SP vs. DNP-HSA); ${ }^{*}<0.05 ;{ }^{* *} P<0.01$. Data were pooled from 3 independent experiments, each of which gave similar results. $n=$ total number of mice per condition. 
A

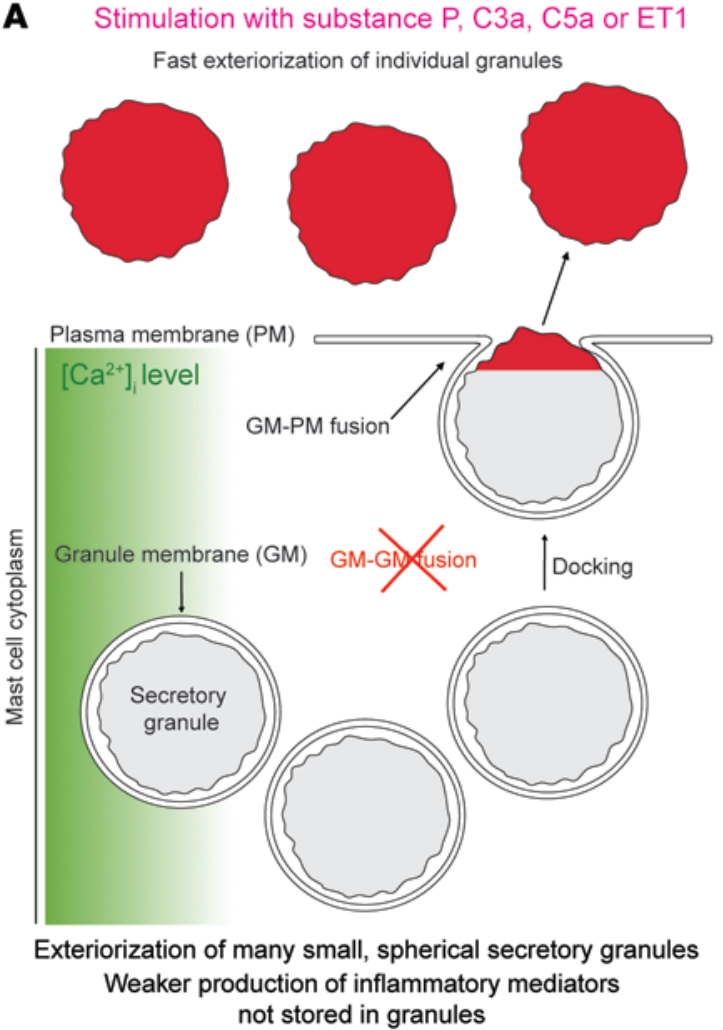

B

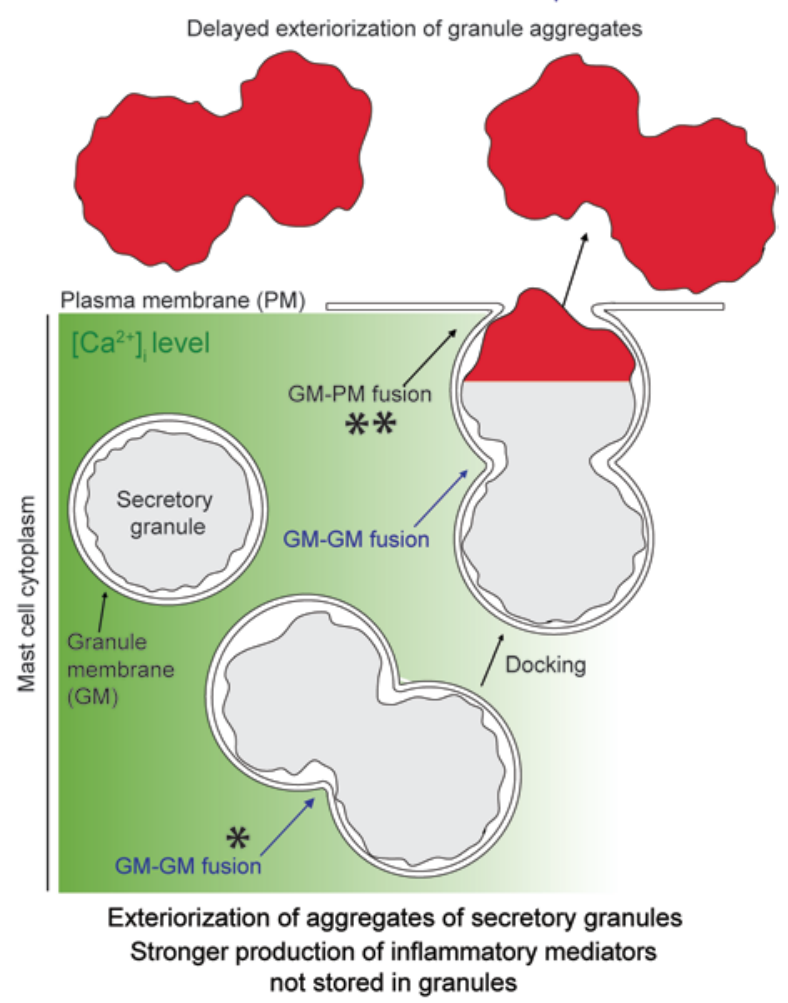

Figure 9. Model of distinct patterns of MC degranulation in response to SP, C3a, C5a, or ET1 versus anti-IgE or IgG immune complexes. (A) Activation with SP, C3a, C5a, or ET1 induces rapid docking of discrete intracellular cytoplasmic granules with the plasma membrane, a process associated with the relatively transient elevation of levels of intracellular calcium ([C $\left.\mathrm{Ca}^{2+}\right]_{\mathrm{i}}$, indicated by a narrow green gradient) and resulting in the fast secretion of small spherical Av.SRho+ secretory granule structures that are likely to represent single granules. (B) Activation via Fc\&RI or Fc $\gamma R$ results in a longer time partition between signaling and secretion, associated with a period of sustained elevation of [Ca $\left.{ }^{2+}\right]_{i}$ levels (indicated by a wide green gradient). During this period, and before granule matrix material can be detected on the cell surface by binding of Av.SRho, some granules undergo deacidification, perhaps reflecting transient fusion of the granule membranes with the plasma membrane (not shown), favoring what may appear to represent, in static images, "intracellular" granule-granule fusion $\left(^{*}\right)$. The fusion of granules with each other and the plasma membrane that occurs during the process of compound exocytosis ${ }^{* *}$ ) results in the formation of heterogeneously shaped granule structures, likely representing aggregates of the matrices of multiple individual granules, that undergo exteriorization (as reflected by the binding of Av.SRho) over a more prolonged period than is seen with SP activation.

the greater enlargement of such DLNs compared with those draining sites of SP injection. It recently has been proposed by the group of Soman Abraham $(25,71)$ that MC granule-associated bioactive molecules (e.g., TNF or IL-12) are protected from dilution and degradation when embedded in the matrix of exteriorized MC granules, and that this initially favors their transport and then the slow solubilization of the cargo content, thus helping to maintain the persistence of the biological signals until they reach their target. It is tempting to speculate that MC compound exocytosis is a mechanism that developed relatively late in evolution, in parallel with the development of antibodies, that permits MCs to generate granule aggregates big enough to constrain the rapid release and/or degradation of preformed bioactive molecules and also to favor their dissemination via lymphatics and/or persistence within the tissues. As proposed by Abraham and colleagues $(25,71)$, the transport of such granule structures within tissues would enable the biodiffusion of their cargo for prolonged periods of time, and therefore facilitate the initiation of distant immune mechanisms that favor the activation of adaptive immune responses.

\section{Methods}

Human primary $M C$ cultures. Human PBCMCs were generated as previously described $(33,34)$. CD $34^{+}$peripheral blood cells were isolated from $2^{\prime} 10^{8}$ peripheral blood mononuclear cells using a Human CD34 Positive Selection Kit (STEMCELL Technologies). PBCMCs were generated from isolated $\mathrm{CD} 34^{+}$cells as described previously in detail (36) and as described in the Supplemental Methods. Before use in experiments, PBCMCs were tested for phenotype by flow cytometry (tryptase, chymase, CD117, FceRI) and function ( $\beta$-hexosaminidase release in response to FceRI cross-linking) at 8-12 weeks. PBCMCs were usually ready for experiments after about 10 weeks in culture.

Statistics. Statistical tests were performed with the software Prism 6 (GraphPad Software). Two-tailed Student's $t$ tests (unpaired or paired) and 2-way ANOVA tests were performed as noted in the respective figure legends. A $P$ value of less than 0.05 was considered statistically significant.

Animal study approval. All animal care and experimentation were conducted in compliance with the guidelines of the National Institutes of Health and with the specific approval of the Institutional Animal Care and Use Committee of Stanford University. 
Other methods are described in Supplemental Methods, which also include images of the full uncut gels used for the panels shown in Figure 3, H-J and Figure 4, E and F.

\section{Author contributions}

NG, EE, and SJG conceived the project. NG, RS, TM, PS, LLR, NC, SV, MT, EE, and SJG were involved in experimental design. NG performed most experiments and compiled the data. RS, TM, PS, LLR, NC, JDH, and EE helped with experiments. All authors participated in analyzing the data and writing or editing the paper.

\section{Acknowledgments}

We thank all members of the Valitutti and Galli laboratories for discussions, and Chen Liu and Mariola Liebersbach for technical assistance. We also thank Andrew Olson and the Stanford Neuroscience Microscopy Service (supported by NIH NS069375), John Perrino and the Cell Science Imaging Facility (supported by ARRA Award 1S10RR026780-01 from the National Center for Research Resources [NCRR]; this article is solely the responsibility of the authors and does not necessarily represent the official views of the NCRR or the NIH), Fatima L'Faqihi (IFR30, Plateau Technique Cytométrie, Toulouse), and Sophie Allart (IFR30, Plateau Technique Imagerie Cellulaire, Toulouse) for technical assistance. N. Gaudenzio is supported by fellowships from the French "Fondation pour la Recherche Médicale FRM" (award SPE20130326582) and the Philippe
Foundation. R. Sibilano is supported by a fellowship from the Lucile Packard Foundation for Children's Health and a Stanford NIH/National Center for Research Resources Clinical and Translational Science Award (UL1-RR025744). T. Marichal is supported by a Marie Curie International Outgoing Fellowship for Career Development: European Union's Seventh Framework Programme (FP7-PEOPLE-2011-IOF), grant 299954. P. Starkl was supported by a Max Kade Fellowship of the Max Kade Foundation and the Austrian Academy of Sciences and a Schroedinger Fellowship of the Austrian Science Fund (FWF), J3399-B21. Laurent L. Reber acknowledges support from the European Commission (Marie Skłodowska-Curie Individual Fellowship H2020-MSCA-IF-2014 656086) and INSERM. This work was supported by grants from the Institut National du Cancer (2012-054), the Agence Nationale de la Recherche (Laboratoire d'Excellence Toulouse Cancer) (to S. Valitutti), the NIH (grants AI023990, CA072074, AI070813, and U19AI104209 to S.J. Galli), and the United States-Israel Binational Science Foundation (grant 2013263 to R. Sagi-Eisenberg, I. Hammel, and S.J. Galli).

Address correspondence to: Eric Espinosa, UMR 1043 INSERM, CPTP CHU Purpan, BP3028, 31024 Toulouse, France. Phone: 0562748304; E-mail: eric.espinosa@inserm.fr. Or to: Stephen J. Galli, 269 Campus Drive, Room 3255b, Stanford, California 943055176, USA. Phone: 650.736.6014; E-mail: sgalli@stanford.edu.
1. Burgoyne RD, Morgan A. Secretory granule exocytosis. Physiol Rev. 2003;83(2):581-632.

2. Metz M, et al. Mast cells can enhance resistance to snake and honeybee venoms. Science. 2006;313(5786):526-530.

3. Schneider LA, Schlenner SM, Feyerabend TB, Wunderlin M, Rodewald HR. Molecular mechanism of mast cell mediated innate defense against endothelin and snake venom sarafotoxin. JExp Med. 2007;204(11):2629-2639.

4. Marichal T, et al. A beneficial role for immunoglobulin $\mathrm{E}$ in host defense against honeybee venom. Immunity. 2013;39(5):963-975.

5. Abraham SN, St John AL. Mast cell-orchestrated immunity to pathogens. Nat Rev Immunol. 2010;10(6):440-452.

6. Stone KD, Prussin C, Metcalfe DD. IgE, mast cells, basophils, and eosinophils. J Allergy Clin Immunol. 2010;125(2 suppl 2):S73-S80.

7. Galli SJ, Tsai M. IgE and mast cells in allergic disease. Nat Med. 2012;18(5):693-704.

8. Galli SJ, Nakae S, Tsai M. Mast cells in the development of adaptive immune responses. Nat Immunol. 2005;6(2):135-142.

9. MacGlashan DW. Self-termination/anergic mechanisms in human basophils and mast cells. Int Arch Allergy Immunol. 2009;150(2):109-121.

10. Azouz NP, Hammel I, Sagi-Eisenberg R. Characterization of mast cell secretory granules and their cell biology. DNA Cell Biol. 2014;33(10):647-651.

11. Turner H, Kinet JP. Signalling through the high-affinity IgE receptor Fc epsilonRI. Nature. 1999;402(6760 suppl):B24-B30.

12. Gilfillan AM, Tkaczyk C. Integrated signalling pathways for mast-cell activation. Nat Rev Immunol. 2006;6(3):218-230.

13. Kalesnikoff J, Galli SJ. New developments in mast cell biology. Nat Immunol. 2008;9(11):1215-1223.

14. Galli SJ, Borregaard N, Wynn TA. Phenotypic and functional plasticity of cells of innate immunity: macrophages, mast cells and neutrophils. Nat Immunol. 2011;12(11):1035-1044.

15. Sibilano R, Frossi B, Pucillo CE. Mast cell activation: a complex interplay of positive and negative signaling pathways. Eur J Immunol. 2014;44(9):2558-2566.

16. Wernersson S, Pejler G. Mast cell secretory granules: armed for battle. Nat Rev Immunol. 2014;14(7):478-494.

17. Maurer M, et al. Mast cells promote homeostasis by limiting endothelin-1-induced toxicity. Nature. 2004;432(7016):512-516.

18. Wang H, Wang HS, Liu ZP. Agents that induce pseudo-allergic reaction. Drug Discov Ther. 2011;5(5):211-219.

19. Schäfer B, et al. Mast cell anaphylatoxin receptor expression can enhance IgE-dependent skin inflammation in mice. J Allergy Clin Immunol. 2013;131(2):541-548.e1.

20. Yano H, Wershil BK, Arizono N, Galli SJ. Substance P-induced augmentation of cutaneous vascular permeability and granulocyte infiltration in mice is mast cell dependent. JClin Invest. 1989;84(4):1276-1286.

21. Verschraegen CF, et al. Phase II study of cetrorelix, a luteinizing hormone-releasing hormone antagonist in patients with platinum-resistant ovarian cancer. Gynecol Oncol. 2003;90(3):552-559.

22. Lumry WR, et al. Randomized placebo-con- trolled trial of the bradykinin $\mathrm{B}_{2}$ receptor antagonist icatibant for the treatment of acute attacks of hereditary angioedema: the FAST-3 trial. Ann Allergy Asthma Immunol. 2011;107(6):529-537.

23. Nishida K, et al. FceRI-mediated mast cell degranulation requires calcium-independent microtubule-dependent translocation of granules to the plasma membrane. JCell Biol. 2005;170(1):115-126.

24. Tiwari N, et al. VAMP-8 segregates mast cell-preformed mediator exocytosis from cytokine trafficking pathways. Blood. 2008;111(7):3665-3674.

25. Kunder CA, et al. Mast cell-derived particles deliver peripheral signals to remote lymph nodes. J Exp Med. 2009;206(11):2455-2467.

26. Cohen R, Corwith K, Holowka D, Baird B. Spatiotemporal resolution of mast cell granule exocytosis reveals correlation with $\mathrm{Ca}^{2+}$ wave initiation. JCell Sci. 2012;125(pt 12):2986-2994.

27. Azouz NP, et al. Rab5 is a novel regulator of mast cell secretory granules: impact on size, cargo, and exocytosis. J Immunol. 2014;192(9):4043-4053.

28. Brochetta C, et al. Munc18-2 and syntaxin 3 control distinct essential steps in mast cell degranulation. JImmunol. 2014;192(1):41-51.

29. Vincent L, et al. Mast cell activation contributes to sickle cell pathobiology and pain in mice. Blood. 2013;122(11):1853-1862.

30. Fujisawa D, et al. Expression of Mas-related gene $\mathrm{X} 2$ on mast cells is upregulated in the skin of patients with severe chronic urticaria. J Allergy Clin Immunol. 2014;134(3):622-633.e9.

31. Tatemoto K, et al. Immunoglobulin E-independent activation of mast cell is mediated by Mrg receptors. Biochem Biophys Res Commun. 2006;349(4):1322-1328. 
32. McNeil BD, et al. Identification of a mast-cell-specific receptor crucial for pseudo-allergic drug reactions. Nature. 2015;519(7542):237-241.

33. Gaudenzio N, Laurent C, Valitutti S, Espinosa E. Human mast cells drive memory $\mathrm{CD} 4^{+} \mathrm{T}$ cells toward an inflammatory IL-22 ${ }^{+}$phenotype. J Allergy Clin Immunol. 2013;131(5):1400-1407.e11.

34. Joulia R, et al. Mast cells form antibody-dependent degranulatory synapse for dedicated secretion and defence. Nat Commun. 2015;6:6174.

35. Dvorak AM. Human mast cells. Adv Anat Embryol Cell Biol. 1989;114:1-107.

36. Subramanian H, Kashem SW, Collington SJ, Qu H, Lambris JD, Ali H. PMX-53 as a dual CD88 antagonist and an agonist for Mas-related gene 2 (MrgX2) in human mast cells. Mol Pharmacol. 2011;79(6):1005-1013.

37. Johnson AR, Moran NC. Selective release of histamine from rat mast cells by compound 48-80 and antigen. Am J Physiol. 1969;216(3):453-459.

38. Dvorak AM, et al. Immunoglobulin E-mediated degranulation of isolated human lung mast cells. Lab Invest. 1985;53(1):45-56.

39. Dvorak AM, Massey W, Warner J, Kissell S, Kagey-Sobotka A, Lichtenstein LM. IgE-mediated anaphylactic degranulation of isolated human skin mast cells. Blood. 1991;77(3):569-578.

40. Williams RM, Webb WW. Single granule $\mathrm{pH}$ cycling in antigen-induced mast cell secretion. J Cell Sci. 2000;113(pt 21):3839-3850.

41. Wollman R, Meyer T. Coordinated oscillations in cortical actin and $\mathrm{Ca}^{2+}$ correlate with cycles of vesicle secretion. Nat Cell Biol. 2012;14(12):1261-1269.

42. Malbec O, et al. Peritoneal cell-derived mast cells: an in vitro model of mature serosal-type mouse mast cells. JImmunol. 2007;178(10):6465-6475.

43. Gaudenzio N, Espagnolle N, Mars LT, Liblau R, Valitutti S, Espinosa E. Cell-cell cooperation at the Thelper cell/mast cell immunological synapse. Blood. 2009;114(24):4979-4988.

44. Lorentz A, Baumann A, Vitte J, Blank U. The SNARE Machinery in Mast Cell Secretion. Front Immunol. 2012;3:143.

45. Guo Z, Turner C, Castle D. Relocation of the t-SNARE SNAP-23 from lamellipodia-like cell surface projections regulates compound exocytosis in mast cells. Cell. 1998;94(4):537-548.

46. Paumet F, et al. Soluble NSF attachment protein receptors (SNAREs) in RBL-2H3 mast cells: functional role of syntaxin 4 in exocytosis and identification of a vesicle-associated membrane protein 8-containing secretory compartment. J Immunol. 2000;164(11):5850-5857.

47. Suzuki K, Verma IM. Phosphorylation of SNAP23 by IкB kinase 2 regulates mast cell degranulation. Cell. 2008;134(3):485-495.

48. Peschke K, et al. IкB kinase 2 is essential for IgE-induced mast cell de novo cytokine production but not for degranulation. Cell Rep. 2014;8(5):1300-1307.

49. Burke JR, et al. BMS-345541 is a highly selective inhibitor of $\mathrm{I} \kappa \mathrm{B}$ kinase that binds at an allosteric site of the enzyme and blocks NF-кBdependent transcription in mice. J Biol Chem. 2003;278(3):1450-1456.

50. MacMaster JF, et al. An inhibitor of I $\kappa$ B kinase, BMS-345541, blocks endothelial cell adhesion molecule expression and reduces the severity of dextran sulfate sodium-induced colitis in mice. Inflamm Res. 2003;52(12):508-511.

51. McIntyre KW, et al. A highly selective inhibitor of I $\mathrm{B}$ kinase, BMS-345541, blocks both joint inflammation and destruction in collagen-induced arthritis in mice. Arthritis Rheum. 2003;48(9):2652-2659.

52. Townsend RM, et al. A highly selective inhibitor of IкB kinase, BMS-345541, augments graft survival mediated by suboptimal immunosuppression in a murine model of cardiac graft rejection. Transplantation. 2004;77(7):1090-1094.

53. Karim ZA, et al. I $\kappa$ kinase phosphorylation of SNAP-23 controls platelet secretion. Blood. 2013;121(22):4567-4574.

54. Hammel I, Lagunoff D, Galli SJ. Regulation of secretory granule size by the precise generation and fusion of unit granules. J Cell Mol Med. 2010;14(7):1904-1916.

55. Scholten J, et al. Mast cell-specific Cre/loxPmediated recombination in vivo. Transgenic Res. 2008;17(2):307-315.

56. Dudeck A, et al. Mast cells are key promoters of contact allergy that mediate the adjuvant effects of haptens. Immunity. 2011;34(6):973-984.

57. Riley JF. Histamine in tissue mast cells. Science. 1953;118(3064):332.

58. Heltianu C, Simionescu M, Simionescu N. Histamine receptors of the microvascular endothelium revealed in situ with a histamine-ferritin conjugate: characteristic high-affinity binding sites in venules. J Cell Biol. 1982;93(2):357-364.

59. McLeod RL, Mingo GG, Kreutner W, Hey JA. Effect of combined histamine $\mathrm{H} 1$ and $\mathrm{H} 3$ receptor blockade on cutaneous microvascular permeability elicited by compound 48/80. Life Sci.
2005;76(16):1787-1794

60. Kunder CA, St John AL, Abraham SN. Mast cell modulation of the vascular and lymphatic endothelium. Blood. 2011;118(20):5383-5393.

61. Wershil BK, Mekori YA, Murakami T, Galli SJ. 125I-fibrin deposition in IgE-dependent immediate hypersensitivity reactions in mouse skin. Demonstration of the role of mast cells using genetically mast cell-deficient mice locally reconstituted with cultured mast cells. J Immunol. 1987;139(8):2605-2614.

62. Grady EF, et al. Substance P mediates inflammatory oedema in acute pancreatitis via activation of the neurokinin-1 receptor in rats and mice. Br JPharmacol. 2000;130(3):505-512.

63. Egawa G, Nakamizo S, Natsuaki Y, Doi H, Miyachi Y, Kabashima K. Intravital analysis of vascular permeability in mice using two-photon microscopy. Sci Rep. 2013;3:1932.

64. Akahoshi M, et al. Mast cell chymase reduces the toxicity of Gila monster venom, scorpion venom, and vasoactive intestinal polypeptide in mice. JClin Invest. 2011;121(10):4180-4191.

65. Gibbs BF, Wierecky J, Welker P, Henz BM, Wolff HH, Grabbe J. Human skin mast cells rapidly release preformed and newly generated TNF-alpha and IL-8 following stimulation with anti-IgE and other secretagogues. Exp Dermatol. 2001;10(5):312-320.

66. Kulka M, Sheen CH, Tancowny BP, Grammer LC, Schleimer RP. Neuropeptides activate human mast cell degranulation and chemokine production. Immunology. 2008;123(3):398-410.

67. Jensen BM, Frandsen PM, Raaby EM, Schiøtz PO, Skov PS, Poulsen LK. Molecular and stimulus-response profiles illustrate heterogeneity between peripheral and cord blood-derived human mast cells. JLeukoc Biol. 2014;95(6):893-901.

68. Suzuki R, et al. Molecular editing of cellular responses by the high-affinity receptor for IgE. Science. 2014;343(6174):1021-1025.

69. Maurer M, Church MK. Inflammatory skin responses induced by icatibant injection are mast cell mediated and attenuated by $\mathrm{H}(1)$-antihistamines. Exp Dermatol. 2012;21(2):154-155.

70. Bochner BS, et al. Interleukin-1 is released at sites of human cutaneous allergic reactions. J Allergy Clin Immunol. 1990;86(6 pt 1):830-839.

71. St John AL, Chan CY, Staats HF, Leong KW, Abraham SN. Synthetic mast-cell granules as adjuvants to promote and polarize immunity in lymph nodes. Nat Mater. 2012;11(3):250-257. 